\section{A aids no Estado de São Paulo. As mudanças no perfil da epidemia e perspectivas da vigilância epidemiológica}

\section{AIDS in the State of São Paulo. Changes in the profile of the epidemic and prospects for epidemiological surveillance}

\author{
Naila Janilde Seabra Santos \\ Doutora em Saúde Pública \\ Gerente da Divisão de Vigilância Epidemiológica \\ CRT-DST-AIDS \\ Secretaria de Estado da Saúde, São Paulo, SP \\ Rua Santa Cruz, n 81, V. Mariana, São Paulo - SP
}

\section{Angela Tayra}

Mestre em Saúde Pública

Diretora Técnica do Serviço de Vigilância Epidemiológica CRT-DST-AIDS

Secretaria de Estado da Saúde, São Paulo, SP

\section{Sara Romera Silva}

Mestre em Saúde Pública

Médica Sanitarista do Serviço de Vigilância Epidemiológica CRT-DST-AIDS

Secretaria de Estado da Saúde, São Paulo, SP

\section{Cassia Maria Buchalla}

Doutora em Saúde Pública

Departamento de Epidemiologia

Faculdade de Saúde Pública

Universidade de São Paulo, São Paulo, SP

Rua Dr. Arnaldo, 715, São Paulo - SP

\section{Ruy Laurenti}

Professor Titular

Departamento de Epidemiologia

Faculdade de Saúde Pública

Universidade de São Paulo, São Paulo, SP

Diretor do Centro Brasileiro de Classificação de Doenças

\section{Resumo}

O HIV, inicialmente vinculado a homens que fazem sexo com homens (HSH), particularmente nos países industrializados e na América Latina, disseminou-se rapidamente entre os diversos segmentos, alcançando mulheres, homens com prática heterossexual e crianças.

A crescente desigualdade entre países desenvolvidos e em desenvolvimento reflete-se, tanto na magnitude da propagação do HIV, quanto na mortalidade por aids. $\mathrm{Na}$ medida em que se acentuam as diferenças de acesso ao tratamento, diminui a mortalidade por aids nos países mais ricos e aumenta nos países mais pobres, exceção feita ao Brasil, um dos poucos países que adotaram a política de distribuição gratuita de antiretrovirais. Aqui, a mortalidade vem apresentando queda acentuada a partir de $1996 \mathrm{e}$ o uso de anti-retrovirais, entre outros, é um dos principais fatores associados a esta diminuição.

No presente artigo foram analisados os dados do Sistema de Vigilância Epidemiológica de DST/aids do Estado de São Paulo, com o intuito de descrever o perfil da epidemia e discutir os termos juvenilização, pauperização, heterossexualização e feminização, introduzidos no discurso sobre a epidemia, para acompanhar as mudanças de seu padrão epidemiológico. Até31/12/2001, no Estado de São Paulo, foram notificados 106.873 casos da doença, o que representa cerca de $50 \%$ do total de notificações do país.

Os maiores coeficientes de incidência aparecem nos indivíduos de 30 a 39 anos, sendo que tanto o número de casos como o de óbitos mostra um ligeiro aumento nas idades mais avançadas, indicando um leve "envelhecimento" da epidemia.

A aids aparece em todas as camadas sociais. Ao longo dos anos tem havido um aumento do número de casos entre pessoas de menor escolaridade, com ocupações menos qualificadas.

O crescimento do número de casos entre homens heterossexuais, junto ao marcante predomínio desta forma de transmis- 
são na população feminina, corrobora a hipótese de heterossexualização da epidemia.

O atual sistema de vigilância epidemiológica de aids é baseado principalmente na notificação de casos e tem sido utilizado como principal fonte de informação para observação das tendências da epidemia e para o planejamento das atividades de prevenção e assistência, assim como para divulgação da doença para a população em geral. Reflete uma situação de vários anos após a infecção ter acontecido, e este intervalo de tempo tende a aumentar em virtude de diversos fatores, tais como a introdução dos anti-retrovirais, entre outros, levando ao aumento do tempo para os casos entrarem no sistema de informação, fazendo com que as informações do sistema atual fiquem cada vez mais distantes da real magnitude da infecção pelo HIV.

Dessa forma, outras estratégias têm sido implementadas para se avaliar as tendências da infecção pelo HIV e para subsidiar novas atividades de prevenção e controle, tais como: a notificação compulsória de gestantes HIV positivas e crianças expostas ao HIV; notificação dos portadores assintomáticos do HIV; aprimoramento da investigação sobre a situação de risco dos casos de HIV/ aids e incorporação do quesito cor/raça na notificação de casos de aids para subsidiar a definição de grupos de risco acrescido e de maior vulnerabilidade; assim como os sistemas de vigilância de segunda geração, que objetivam identificar as tendências do comportamento e de prevalência da infecção.

Palavras chave: HIV/AIDS. Heterossexualização. Feminização. Pauperização. Envelhecimento. Vigilância epidemiológica.

\section{Abstract}

HIV, initially associated with men who have sex with men, mainly in industrialized countries and Latin America, spread quickly reaching women, heterosexual men and children.

The increasing inequalities between industrialized and developing countries become apparent in rates of HIV infection and AIDS mortality. As the differences in access to AIDS treatment grow, mortality due to AIDS decreases in richer countries and increases in poorer countries, with the exception of Brazil, one of the few countries in the world that has free distribution of combination antiretroviral therapy. In Brazil, AIDS mortality has been falling since 1996, and has been substantially affected by access to AIDS treatment.

In this paper, data on STD and AIDS, collected from the epidemiological surveillance system of the State of São Paulo, were analyzed with the purpose of describing the profile of the AIDS epidemic, and discussing the way this disease affects women and young, poor and heterosexual persons. Until 12/31/2001, 106,873 cases were reported in the State of São Paulo, which was about $50 \%$ of the cases reported in the country as a whole.

The highest incidence rates occur among individuals between 30 and 39 years of age. The number of cases and deaths has grown slightly in older individuals. AIDS affects all, the rich and the poor, but in past years it has been increasing in individuals with fewer years of education and non-qualified occupations.

The increase in cases among heterosexual men and the fact that this mode of exposure is the most frequent among women corroborates the importance of heterosexual transmission in the AIDS epidemic.

The STD and AIDS epidemiological surveillance system in Brazil is based mainly upon cases reported, and it has been used as the main source of information on the AIDS epidemic. These epidemiological surveillance 
data support the study of trends, prevention and care planning, and provides the means to promote information on AIDS to the population. Such data reflect the status of HIV several years after infection occurred, and this gap tends to grow due to many factors, such as antiretroviral therapy and others. In this manner, data take longer to enter the information system, and therefore, such information becomes increasingly distant from the actual magnitude of HIV infection.

Other strategies for acknowledging trends of HIV infections are necessary in order to make its control more effective. Examples are reporting HIV infection in pregnant women and their children, and in people living with HIV (without AIDS); and implementing information from case reports, by introducing data on color or race, investigating forms of exposure to HIV infection, defining risks and vulnerability of population groups and by using the strategies of second generation epidemiological surveillance.

Keywords: HIV/AIDS. Heterosexuals. Women. Impoverishment. The elderly. Epidemiological Surveillance.

\section{Introdução}

Os primeiros casos da Síndrome da Imunodeficiência Adquirida-AIDS, ou aids, como se denomina atualmente no Brasil, foram descritos nos Estados Unidos da América em $1981^{1,2} \mathrm{e}$, ainda que naquele momento não se conhecesse sua causa, a nota editorial que acompanhava a publicação sugeria "uma disfunção da imunidade celular relacionada a uma exposição comum" e "uma patologia adquirida por contato sexual" ${ }^{3} \mathrm{Na}$ primeira metade da década de 80 , o vírus da imunodeficiência humana (HIV) foi isolado e relacionado à aids ${ }^{4,5}$.

Embora inicialmente vinculado aos homens que fazem sexo com outros homens (HSH), particularmente nos países industrializados e na América Latina, o HIV se disseminou rapidamente entre os diversos segmentos da sociedade, alcançando paulatinamente mulheres, crianças e homens com prática heterossexual.

Essa disseminação se deu não só, embora principalmente, pela via sexual ${ }^{6-8}$, mas também pela via sangüínea, através do compartilhamento de seringas e agulhas por usuários de drogas injetáveis, em transfusões de sangue e hemoderivados e, ainda, pela via materno-infantil (transmissão da mãe para a criança na gestação, parto ou durante $\mathrm{o}$ aleitamento materno), na medida em que as mulheres foram sendo atingidas.

Pode-se perceber diferentes padrões de transmissão da infecção pelo HIV obedecendo a distintas formas de organização social ${ }^{9-}$ ${ }^{12}$. No continente africano, as principais formas de transmissão do HIV sempre foram as relações heterossexuais e a transmissão materno-infantil. Na Europa Ocidental, EUA e em vários países da América Latina, apesar da relevância dos HSH, particularmente nos primórdios da epidemia, o compartilhamento de seringas e agulhas por usuários de drogas injetáveis surgiu como uma importante forma de disseminação do HIV.

No Estado de São Paulo, os padrões de transmissão são distintos nas diversas regiões do Estado $^{13}$ e o compartilhamento de seringas e agulhas pelo uso de drogas injetá- 
veis destaca-se enquanto forma de disseminação da infecção pelo HIV, tendo se constituído em um modo bastante eficiente de atingir a população heterossexual, através da alta porcentagem de indivíduos que se infectaram pela parceria sexual com usuários(as) de drogas injetáveis ${ }^{14,15}$.

Assim, se no início a infecção pelo HIV parecia limitar-se a determinados "grupos de risco" compostos por homossexuais, hemofílicos e usuários de drogas, com o correr do tempo ela foi buscando caminhos, tomando espaços na população geral ${ }^{7,16} \mathrm{e}$ "socializando" a sua possibilidade de ocorrência, que antes parecia restrita aos "diferentes e famosos”, passando progressivamente por um processo de pauperização $0^{9,17-21}$ e feminização ${ }^{10,14,22-24}$ da epidemia.

Em poucos anos, a infecção pelo HIV transformou-se em uma pandemia.

Tendo atingido particularmente a população de adultos jovens em todo o mundo, a aids foi responsável por milhares de Anos Potenciais de Vida Perdidos (APVP) do final dos anos 80 a meados dos 90, levando a uma diminuição significativa da esperança de vida em várias localidades e aparecendo como a principal causa de mortalidade para a população entre 15 e 49 anos em inúmeros países, inclusive no Brasil. No Município de São Paulo, em 1995, a aids foi a segunda causa na hierarquização dos APVP para os homens e a primeira para as mulheres ${ }^{25}$.

Entretanto, numerosos países, entre os quais o Brasil, têm apresentado nos últimos anos uma tendência de queda dos óbitos por esta doença, com significativa mudança no perfil de mortalidade da epidemia.

A crescente desigualdade entre os países desenvolvidos e em desenvolvimento refletese tanto na magnitude da propagação do HIV quanto na mortalidade por aids. Na medida em que se acentuam as diferenças de acesso ao tratamento, a mortalidade por aids diminui nos países mais ricos e aumenta nos países mais pobres ${ }^{26}$, exceção feita ao Brasil, que é um dos poucos países do mundo que adotaram a política de distribuição gratuita de antiretrovirais aos portadores de HIV/aids.

No Brasil, os primeiros casos de aids foram diagnosticados em 1982 e, apesar da importância relativa da região Sudeste na epidemia, a aids já foi notificada em todos os estados brasileiros. Suas dimensões continentais, fazem com que este país apresente diferenças regionais importantes no que concerne ao perfil da epidemia.

A epidemia se iniciou de forma semelhante na maioria da regiões brasileiras, na população masculina de homens que fazem sexo com homens; e a transfusão de sangue e hemoderivados constou, na segunda metade da década de 80, como categoria de exposição em inúmeros casos notificados em todo o país, caindo drasticamente após a medidas implantadas em função da legislação de controle do sangue e hemoderivados no que diz respeito à infecção pelo HIV, instituída no Brasil em 198827. O uso de drogas injetáveis, entretanto, adquiriu particular relevância enquanto categoria de exposição ao HIV nas regiões centro-oeste, sul e sudeste ${ }^{28}$, sendo muito importante nesta última região, especialmente no Estado de São Paulo, surgindo como um fator de diferenciação nos padrões de transmissão do HIV no país.

Desde o início da epidemia até 30 de setembro de 2001, 222.356 casos foram notificados no Brasil, sendo 158.685 em homens adultos, 55.824 em mulheres adultas e 7.847 em menores de 13 anos de idade ${ }^{8}$.

Vale ressaltar que as estatísticas de casos notificados no país referem-se apenas ao número de indivíduos que desenvolveram aids e não ao número de indivíduos soropositivos para o HIV (no Brasil apenas a aids é de notificação compulsória*). O Brasil tem utilizado, até hoje, os resultados dos estudos

\footnotetext{
* Existem alguns critérios, não excludentes, que definem um caso de aids em adultos para fins de notificação no Brasil: Critério CDC, que pressupõe a presença de pelo menos uma doença oportunista ou uma contagem de células CD4 menor de 350 cels/mm ${ }^{3}$ em indivíduo soropositivo para o HIV; Critério Rio de Janeiro/Caracas, que se baseia na pontuação de sinais e sintomas característicos da aids e fecha o caso quando um indivíduo HIV positivo somar 10 pontos; Critério ARC+Óbito, quando um indivíduo HIV positivo com alguns sinais e sintomas associados à aids evolui para óbito sem entrar em um dos critérios de definição anteriores; e os critérios excepcionais, utilizados via de regra para pacientes dos quais se obtêm a informação do caso após o seu óbito ${ }^{30}$. Para crianças se aplicam critérios semelhantes, sendo que as faixas consideradas de contagem de células CD4 são diferentes nas diversas faixas etárias e o critério de pontuação Rio de Janeiro/Caracas não se aplica a crianças, sendo este substituído por um critério que define Sinais Maiores e Menores, e são necessários 2 sinais maiores ou 1 sinal maior e 2 menores para definir um caso por este critério ${ }^{31}$.
} 
de clínicas-sentinela e a soroprevalência para o HIV em populações específicas para estimar o número de indivíduos HIV positivos existentes no país, que se calcula estar em torno de $0,8 \%$ da população masculina, $0,47 \%$ da feminina e $0,6 \%$ da população to$\mathrm{tal}^{29}$.

A epidemia de aids é hoje um grande problema de saúde pública no Brasil e, embora até a atualidade haja muito mais casos notificados em indivíduos do sexo masculino, a velocidade de crescimento da epidemia é, como em outros países, muito maior entre as mulheres do que entre os homens.

Também no Brasil, a aids é uma das principais causas de mortalidade em adultos jovens. No entanto, apesar das condições socioeconômicas pouco privilegiadas da maioria da população, a mortalidade vem apresentando uma queda acentuada a partir de 1996, sendo o uso de anti-retrovirais um dos principais fatores associados a esta diminuição, além da descentralização do atendimento, que possibilita um diagnóstico mais precoce e a intervenção adequada sobre as infecções oportunistas, o que altera consideravelmente a sobrevida dos pacientes. Não obstante, nos dois últimos anos, essa queda está apresentando uma visível desaceleração.

É fundamental observar os diversos fatores capazes de influenciar a qualidade e o tempo de vida dos indivíduos com HIV/aids. O acompanhamento laboratorial e o manejo clínico cuidadoso e adequado dos indivíduos HIV positivos por clínicos treinados e eficientes, a quimioprofilaxia e tratamento de determinadas infecções oportunistas que aumentam o risco de evolução fatal ${ }^{32,33}$, a compreensão dos fatores constitucionais e o acompanhamento psicológico dos pacientes $^{34}$, são todos aspectos fundamentais a serem considerados, sem os quais, a administração pura e simples da terapêutica antiretroviral não será suficiente para mudar o prognóstico da infecção pelo HIV. Observese que, mesmo antes da instituição desta terapêutica, a sobrevida dos pacientes já era maior nos países industrializados do que nos países em desenvolvimento.
Os avanços da terapia anti-retroviral e as tendências atuais da epidemia, concentrando-se em países e populações mais socialmente vulneráveis ${ }^{35}$, devem levar a novas reflexões sobre as formas de abordagem do HIV/aids sob suas diversas nuanças, que vão desde o manejo clínico e psicológico dos pacientes já infectados, diante da possibilidade de prolongamento de suas vidas na condição de soropositivos assintomáticos para o HIV, até a implementação e manutenção de políticas públicas de prevenção e assistência cada vez mais eficientes, com o desafio e a obrigação moral e ética de tornálas acessíveis a toda a população ${ }^{36}$.

Tudo isto remete à necessidade de aprimoramento da vigilância epidemiológica e seus sistemas de informação, para que estes possam continuar produzindo um conhecimento que subsidie o planejamento e as ações programáticas na área de atuação de HIV/aids .

\section{Perfil da epidemia de aids no Estado de São Paulo}

Foram analisados os dados obtidos através do Sistema de Vigilância Epidemiológica de DST/aids do Estado de São Paulo (ESP), com o intuito de descrever o perfil da epidemia neste Estado e discutir os termos juvenilização, pauperização, heterossexualização e feminização que foram sendo introduzidos no discurso desta epidemia, na tentativa de acompanhar as mudanças de seus padrões epidemiológicos.

O Estado de São Paulo (ESP) tem sido, desde os primórdios da epidemia, responsável por cerca de $50 \%$ do total de notificações do país, sendo o Município de São Paulo o que apresenta o maior número absoluto de casos de aids no Brasil, como seria de se esperar, dada a sua densidade populacional e suas demais características de grande centro urbano.

O número de casos de aids no Estado de São Paulo com data de diagnóstico e de notificação até 31/12/2001 é de 106.873 casos e destes $44 \%$ (47.047 casos) são residentes no Município de São Paulo ${ }^{13}$. 
No início da epidemia no Estado, a imensa maioria dos casos ocorria em homens, e os coeficientes de incidência no sexo masculino eram expressivamente maiores do que no sexo feminino, mas houve um aumento tão expressivo no número de casos em mulheres que a relação masculino/feminino, que em 1984 era de 27/1, aproximase de 2/ 1 em 1997 e se mantém neste nível, apresentando, a partir de então, ligeira queda ano a ano (Tabela 1 e figura 1) . Os coeficientes de incidência obedecem a um padrão semelhante ao de número de casos, isto é, aumentam até 1995/1996 para os homens e para o total de casos e até 1998 para a população feminina.
Apesar do aumento do número absoluto de casos até 1996, o ritmo de crescimento diminui gradativamente nos anos 90 , especificamente às custas da população masculina.

É precoce avaliar o impacto desta diminuição, pois é sabido que há um atraso muito grande nas notificações e conseqüente demora na chegada da informação ao nível central do Sistema de Vigilância Epidemiológica. Sendo assim, o decréscimo percebido nos anos de 1999 e 2000 não pode ainda ser considerado como real, uma vez que tem havido, ao longo do tempo, inclusão de casos novos em anos muito anteriores à chegada das notificações, sendo essa inclusão mais significativa nos anos mais recentes.

Tabela 1 - Casos notificados de aids, coeficiente de incidência* $(\mathrm{Cl})$ por por 100.000 habitantes segundo ano de diagnóstico, sexo e razão de sexo, estado de são paulo, 1980-2001 (**)

Table 1 - Aids Cases Reported, Incidence Coefficient* (IC) per 100,000 inhabitants, according to year of diagnosis, gender and gender ratio, State of São Paulo, $1980-2001$ ( $\left.^{* *}\right)$

\begin{tabular}{|c|c|c|c|c|c|c|c|c|}
\hline \multirow{3}{*}{$\begin{array}{c}\text { Ano de } \\
\text { Diagnóstico }\end{array}$} & \multicolumn{4}{|c|}{ sexo } & & & \multirow{3}{*}{ razão de sexo } & \multirow{3}{*}{$\%$} \\
\hline & \multicolumn{2}{|c|}{ masculino } & \multicolumn{2}{|c|}{ feminino } & \multicolumn{2}{|c|}{ total } & & \\
\hline & casos & $\mathrm{Cl}$ & casos & $\mathrm{Cl}$ & casos & $\mathrm{Cl}$ & & \\
\hline 1980 & 1 & 0 & - & - & 1 & 0.00 & - & - \\
\hline 1981 & - & - & - & - & - & - & - & - \\
\hline 1982 & 8 & 0.06 & - & - & 8 & 0.03 & - & - \\
\hline 1983 & 24 & 0.18 & 2 & 0.02 & 26 & 0.10 & $12 / 1$ & 12.00 \\
\hline 1984 & 80 & 0.59 & 3 & 0.02 & 83 & 0.31 & $27 / 1$ & 26.67 \\
\hline 1985 & 326 & 2.36 & 12 & 0.09 & 338 & 1.22 & $27 / 1$ & 27.17 \\
\hline 1986 & 585 & 4.16 & 31 & 0.22 & 616 & 2.18 & $19 / 1$ & 18.87 \\
\hline 1987 & 1363 & 9.50 & 163 & 1.12 & 1526 & 5.28 & $9 / 1$ & 8.36 \\
\hline 1988 & 2164 & 14.78 & 367 & 2.47 & 2531 & 8.57 & $6 / 1$ & 5.90 \\
\hline 1989 & 2929 & 19.62 & 507 & 3.33 & 3436 & 11.40 & $6 / 1$ & 5.78 \\
\hline 1990 & 4224 & 27.73 & 786 & 5.05 & 5010 & 16.28 & $5,5 / 1$ & 5.37 \\
\hline 1991 & 5349 & 34.42 & 1166 & 7.34 & 6515 & 20.72 & $5 / 1$ & 4.59 \\
\hline 1992 & 6455 & 40.84 & 1664 & 10.27 & 8119 & 25.36 & $4 / 1$ & 3.88 \\
\hline 1993 & 6739 & 42.03 & 1914 & 11.61 & 8653 & 26.61 & $3,5 / 1$ & 3.52 \\
\hline 1994 & 6837 & 42.02 & 2098 & 12.51 & 8935 & 27.04 & $3,5 / 1$ & 3.26 \\
\hline 1995 & 7368 & 44.64 & 2613 & 15.32 & 9981 & 29.60 & $3 / 1$ & 2.82 \\
\hline 1996 & 7492 & 44.76 & 3033 & 17.49 & 10525 & 30.89 & $3 / 1$ & 2.47 \\
\hline 1997 & 6912 & 40.52 & 3272 & 18.67 & 10184 & 29.45 & $2 / 1$ & 2.11 \\
\hline 1998 & 7026 & 40.57 & 3589 & 20.15 & 10615 & 30.22 & $2 / 1$ & 1.96 \\
\hline 1999 & 5879 & 33.53 & 3011 & 16.68 & 8890 & 24.98 & $2 / 1$ & 1.95 \\
\hline 2000 & 5002 & - & 2631 & - & 7633 & 21.00 & $2 / 1$ & 1.90 \\
\hline 2001 & 2135 & - & 1113 & - & 3248 & - & $2 / 1$ & 1.92 \\
\hline TOTAL & 78,898 & 27,975 & 106,873 & $3 / 1$ & 2.82 & & & \\
\hline
\end{tabular}

Fonte: SINAN- Vigilância Epidemiológica - Programa Estadual DST/Aids-SP; (*) Utilizada projeção populacional da Fundação SEADE; (**) Dados preliminares, sujeitos a revisão mensal, até 31/12/01.

Source: SINAN - Disease Surveillance - STD/AIDS State Program; Fundação SEADE's populational projection; Preliminary data, subject to monthly revisions, until 12/ 31/01. 


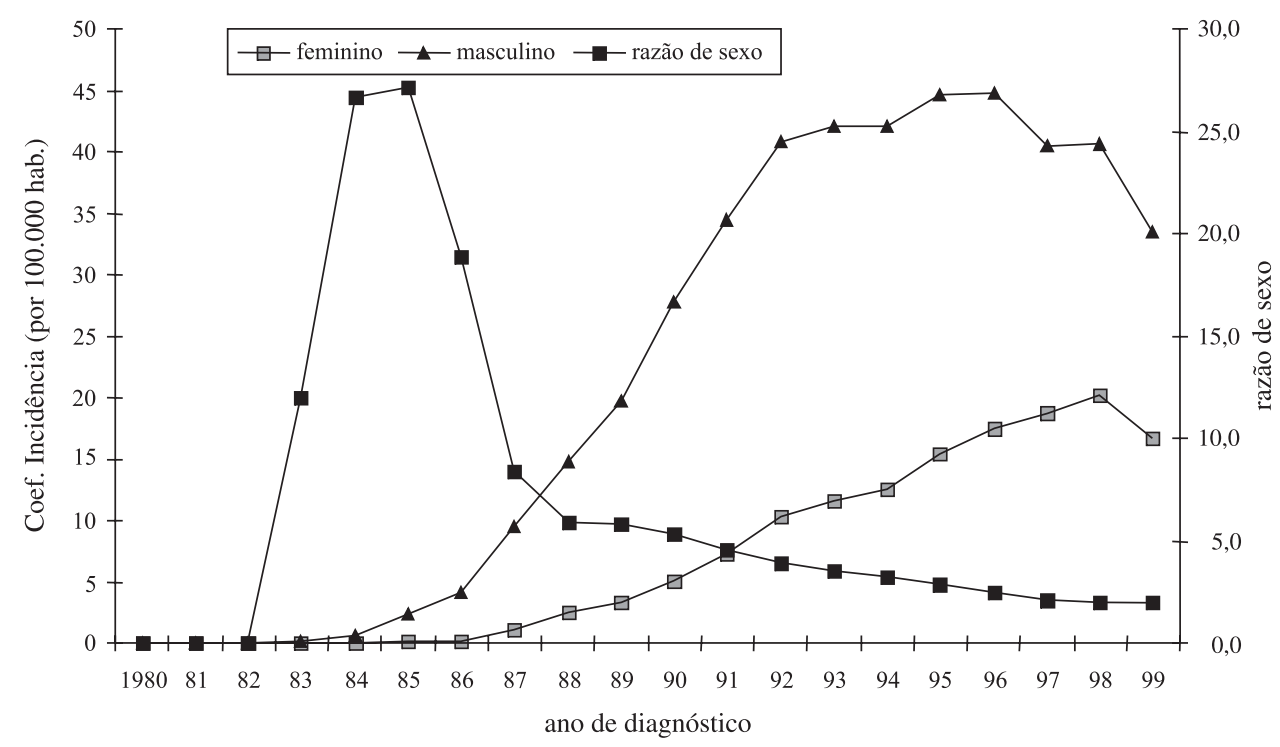

Fonte: SINAN - Vigilância Epidemiológica - Programa Estadual DST/Aids (*dados até 30/12/2001)

Source: SINAN - Disease Surveillance - STD/AIDS State Program ( ${ }^{*}$ data until 12/30/2001)

Figura 1 - Coeficiente de Incidência de Aids segundo sexo, razão de sexo e ano de diagnóstico, Estado de São Paulo, 1980 a 1999*

Figure 1 - AIDS incidence coefficient according to gender, gender ratio and year of diagnosis, State of São Paulo, 1980 to $1999^{*}$

A transmissão por via sexual tem sido a mais importante, tanto em homens quanto em mulheres, sendo responsável por $50 \%$ do total de casos, seguida pela transmissão por via sangüínea, responsável por aproximadamente $30 \%$ dos casos. A via mais importante de transmissão sangüínea, em ambos os sexos, é o uso de drogas injetáveis. Pode-se observar nítida queda da transmissão por transfusão sangüínea, em ambos os sexos e em homens hemofílicos depois de 1989; ressalte-se que a legislação de controle do sangue e hemoderivados, que diz respeito à infecção pelo HIV, foi instituída no Estado de São Paulo em $1986^{36}$, anteriormente a legislação federal.

Há um predomínio muito marcado das categorias homossexual e bissexual no início da epidemia e, até 1987, elas representavam juntas mais de $50 \%$ dos casos masculinos. Nesse ano o uso de drogas injetáveis desponta como uma categoria de transmissão importante para os casos de AIDS e passa a representar cerca de um terço dos casos até meados da década de 1990, quando a proporção da categoria heterossexual aumenta sensivelmente sua importância no cenário da epidemia masculina, sendo, atualmente, o uso de drogas injetáveis e a categoria heterossexual, as situações de risco mais prevalentes entre os homens (Tabela 2).

Saliente-se que na hierarquização de risco utilizada pela VE de DST/aids, a categoria heterossexual só é considerada como forma de transmissão do HIV se o indivíduo não tiver prática bissexual e não referir nenhum risco de transmissão sangüínea. Mesmo assim, imagina-se que este número esteja superestimado, e a importância relativa dos bissexuais na epidemia esteja subestimada, pois no Brasil, a questão da bissexualidade é muitas vezes camuflada por questões morais e culturais, e os indivíduos tendem a esconder este fato, ressaltando suas relações heterossexuais ${ }^{37,38}$. O aumento da proporção de indivíduos heterossexuais pode ser explicado também pelo aumento do número de mulheres infectadas na população. 


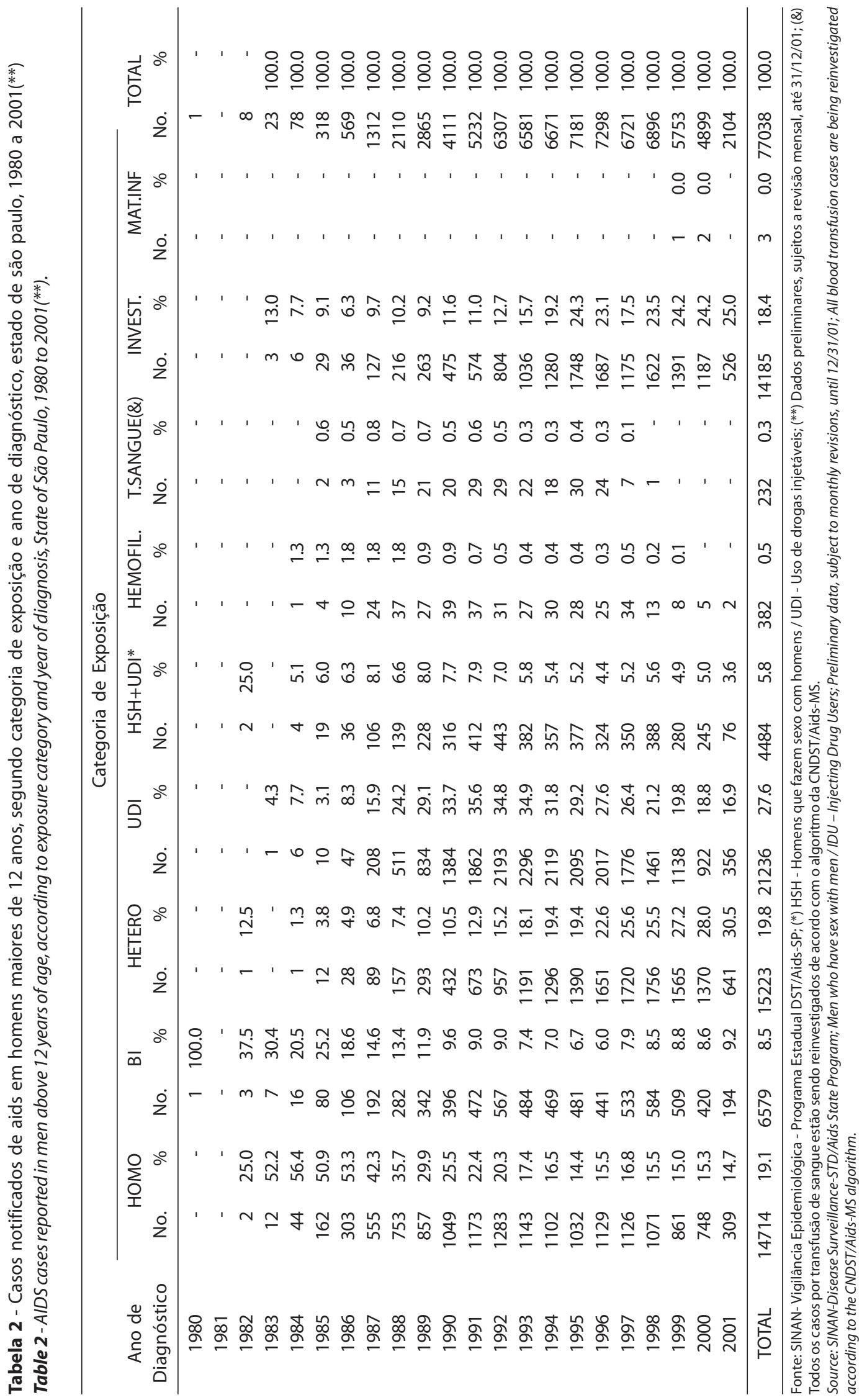


Quando analisamos a evolução da epidemia no sexo feminino (Tabela 3), no Estado de São Paulo, observamos três fases distintas em termos de maior risco para a infecção pelo HIV. A primeira fase se estendeu até 1986, quando a transmissão pela via sexual era a mais importante, sendo, naquele momento, as parcerias com homens bissexuais e transfundidos as mais freqüentes. Nesse período era importante, também, a transmissão pela transfusão sangüínea. A segunda fase compreendeu o período de 1987 a 1990, quando prevaleceram os casos pelo uso de drogas injetáveis; e a terceira fase, de 1991 até o presente, quando a prática hete- rossexual voltou a ser a principal situação de risco descrita entre os casos de aids em mulheres, sendo agora a parceria sexual com homens usuários de drogas injetáveis a mais relevante (Tabela 3).

Há ainda o primeiro caso confirmado de aids por acidente profissional, notificado no Brasil, que é feminino.

Em trabalho realizado com 2.759 casos de mulheres notificados no Estado de São Paulo (ESP), com diagnóstico até julho de 1992, 34,6\% das mulheres que referiram como situação de risco principal a prática sexual, eram parceiras de homens UDI ${ }^{14}$. Pelos dados obtidos através da Vigilância

Tabela 3 - Casos notificados de aids em mulheres maiores de 12 anos, segundo categoria de exposição e ano de diagnóstico, Estado de São Paulo, 1980-2001(*)

Table 3 - Aids cases reported in women over 12 years of age, according to exposure category and year of diagnosis, State of São Paulo, 1980-2001(*)

\begin{tabular}{|c|c|c|c|c|c|c|c|c|c|c|c|c|}
\hline \multirow{3}{*}{$\begin{array}{c}\text { Ano de } \\
\text { Diagnóstico }\end{array}$} & \multicolumn{10}{|c|}{ Categoria de Exposição } & & \\
\hline & \multicolumn{2}{|c|}{ Hetero } & \multicolumn{2}{|c|}{ UDI } & \multicolumn{2}{|c|}{ T.sangue(\&) } & \multicolumn{2}{|c|}{ Mat. Inf. } & \multicolumn{2}{|c|}{ Invest. } & \multicolumn{2}{|c|}{ Total } \\
\hline & No. & $\%$ & No. & $\%$ & No. & $\%$ & No. & $\%$ & No. & $\%$ & No. & $\%$ \\
\hline 1980 & - & - & - & - & - & - & - & - & - & - & - & - \\
\hline 1981 & - & - & - & - & - & - & - & - & - & - & - & - \\
\hline 1982 & - & - & - & - & - & - & - & - & - & - & - & - \\
\hline 1983 & 1 & 50.0 & 1 & 50.0 & - & - & - & - & - & - & 2 & 100.0 \\
\hline 1984 & 1 & - & - & - & - & - & - & - & 2 & 66.7 & 3 & 100.0 \\
\hline 1985 & 6 & 54.5 & 1 & - & 1 & 9.1 & - & - & 3 & 27.3 & 11 & 100.0 \\
\hline 1986 & 13 & 46.4 & 8 & 28.6 & 2 & 7.1 & - & - & 5 & 17.9 & 28 & 100.0 \\
\hline 1987 & 45 & 30.4 & 82 & 55.4 & 6 & 4.1 & - & - & 15 & 10.1 & 148 & 100.0 \\
\hline 1988 & 105 & 33.7 & 158 & 50.6 & 11 & 3.5 & - & - & 38 & 12.2 & 312 & 100.0 \\
\hline 1989 & 164 & 36.8 & 217 & 48.7 & 15 & 3.4 & - & - & 50 & 11.2 & 446 & 100.0 \\
\hline 1990 & 309 & 43.5 & 318 & 44.7 & 14 & 2.0 & - & - & 70 & 9.8 & 711 & 100.0 \\
\hline 1991 & 483 & 45.7 & 404 & 38.2 & 24 & 2.3 & - & - & 146 & 13.8 & 1057 & 100.0 \\
\hline 1992 & 823 & 53.5 & 526 & 34.2 & 25 & 1.6 & - & - & 164 & 10.7 & 1538 & 100.0 \\
\hline 1993 & 1027 & 57.7 & 490 & 27.5 & 20 & 1.1 & - & - & 244 & 13.7 & 1781 & 100.0 \\
\hline 1994 & 1218 & 62.2 & 436 & 22.3 & 13 & 0.7 & - & - & 290 & 14.8 & 1957 & 100.0 \\
\hline 1995 & 1480 & 61.4 & 413 & 17.1 & 20 & 0.8 & - & - & 498 & 20.7 & 2411 & 100.0 \\
\hline 1996 & 1810 & 63.9 & 469 & 16.6 & 18 & 0.6 & - & - & 534 & 18.9 & 2832 & 100.0 \\
\hline 1997 & 2168 & 70.1 & 428 & 13.8 & 5 & 0.2 & 1 & - & 489 & 15.8 & 3091 & 100.0 \\
\hline 1998 & 2253 & 65.2 & 390 & 11.3 & 2 & 0.1 & 1 & 0.0 & 807 & 23.4 & 3453 & 100.0 \\
\hline 1999 & 1896 & 65.9 & 277 & 9.6 & - & - & - & - & 703 & 24.4 & 2876 & 100.0 \\
\hline 2000 & 1629 & 64.2 & 192 & 7.6 & 1 & - & - & - & 717 & 28.2 & 2539 & 100.0 \\
\hline 2001 & 721 & 66.6 & 81 & 7.5 & - & - & 1 & 0.1 & 280 & 25.9 & 1083 & 100.0 \\
\hline TOTAL & 16152 & 61.5 & 4891 & 18.6 & 177 & 0.7 & 3 & 0.0 & 5055 & 19.2 & 26279 & 100.0 \\
\hline
\end{tabular}

Fonte: SINAN-Vigilância Epidemiológica - Programa Estadual DST/Aids-SP; (**) Dados preliminares, sujeitos a revisão mensal, até 31/12/01; Foi confirmado um caso de exposição por acidente profissional em 1996; (\&) Todos os casos por transfusão de sangue estão sendo reinvestigados de acordo com o algoritmo da CNDST/Aids-MS."

Source: SINAN - Disease Surveillance - STD/AIDS State Program-SP; (**) Preliminary data subject to monthly review, up to 12/31/01; A case due to professional exposure was confirmed for 1996; (\&) All cases of blood transfusion are being reinvestigated according to the CNDST/Aids-MS algorhythm." 
Epidemiológica de DST/aids do Estado de São Paulo, entre todas as mulheres notificadas com aids no Estado, com categoria de exposição heterossexual, do início da epidemia até 1997, 40,1\% referiram parceria sexual com UDI. Em dezembro de 2001, a proporção de mulheres heterossexuais com parceiros usuários de drogas, desde o início da epidemia, foi de $31,7 \%$.

Na Tabela 4 e na Figura 2 podemos observar a proporção do tipo de parcerias entre o total de casos notificados em homens e mulheres com categoria de exposição heterossexual. Note-se que a multiplicidade de parceiros é relatada muito mais freqüentemente pelos homens, e que a parceria com usuários de drogas injetáveis é o tipo mais freqüente entre as mulheres, seguida pela multiplicidade de parceiros.

Estes dados reiteram a observação de que o uso de drogas injetáveis constituiu em importante fator de disseminação da epidemia de aids em nosso meio, particularmente para a população feminina, quer pela transmissão sangüínea entre os usuários de drogas injetáveis, quer pela parceria sexual com os mesmos.

Atualmente, tomando-se o total de casos femininos desde 1983, observa-se que a maioria das mulheres se contaminou pelas relações heterossexuais, sendo o uso de drogas injetáveis a segunda categoria de transmissão mais prevalente entre os casos femininos notificados.

$\mathrm{O}$ aumento do número de casos em mulheres traz, como conseqüência, um maior número de casos em crianças pela transmissão materno-infantil. Esta forma de transmissão é responsável por mais de $80 \%$ dos casos notificados em menores de 13 anos de idade (Tabela 5).

Ressalte-se que, em um momento anterior, já foi observado um resultado bastante satisfatório na redução da infecção pelo HIV através das transfusões sangüíneas e de hemoderivados, devido à adoção de medidas então preconizadas para o controle da qualidade do sangue transfundido. Analogamente, a transmissão vertical pode ser drasticamente reduzida, desde que a gestante e seu concepto tenham o diagnóstico da infecção pelo HIV realizado a tempo e sejam instituídas, também em tempo hábil, as medidas profiláticas hoje disponíveis.

Embora em número muito pequeno, aparecem casos de aids em menores de 13 anos devidos à transmissão sexual (3 casos) e uso de drogas injetáveis (6 casos). Ainda que estes casos não tenham impacto em relação ao total da epidemia, têm uma impor-

Tabela 4 - Casos notificados de aids com categoria de transmissão heterossexual, segundo tipo de parceria sexual e sexo, no estado de são paulo, 1980 a $2001(* *)$.

Table 4 - Aids Cases reported as heterosexual transmission, according to type of sexual partnership and gender. State of São Paulo, 1980 to 2001

\begin{tabular}{lrrrr}
\hline & \multicolumn{3}{c}{ Sexo } \\
\cline { 2 - 5 } Tipo de Parceria & \multicolumn{2}{c}{ Masculino } & \multicolumn{2}{c}{ Feminino } \\
& No. & $\%$ & 3924 & 24.30 \\
\hline Múltipla parceria & 9933 & 65.10 & 5120 & 31.70 \\
Parceiro UDI & 950 & 6.20 & 2259 & 14.00 \\
Parc. Múltiplos Parceiros & 1025 & 6.70 & 201 & 1.20 \\
Parc.Transfundido & 184 & 1.20 & 73 & 0.50 \\
Parc. Hemofílico & - & - & 1137 & 7.00 \\
Parc.Bissexual & - & - & 3441 & 21.30 \\
Outras parcerias & 3175 & 20.80 & 16155 & 100.00 \\
\hline Total & 15267 & 100.00 & & \\
\hline
\end{tabular}

Fonte: SINAN- Vigilância Epidemiológica - Programa Estadual DST/Aids-SP; $\left({ }^{* *}\right)$ Dados preliminares, sujeitos a revisão mensal, até $31 / 12 / 01$.

Source:SINAN-Disease Surveillance-STD/Aids State Program, SP; Data, subject to monthly revision, until 12/31/01 


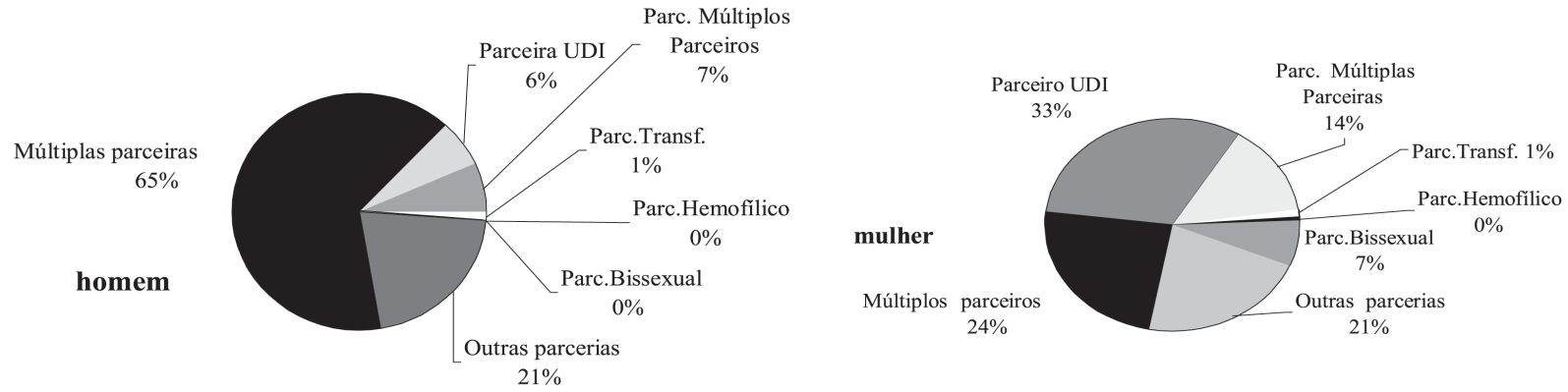

Fonte: Vigilância Epidemiológica - Programa Estadual DST/Aids ( ${ }^{*}$ dados até 30/12/01)

Source: Disease Surveillance - STD/Aids State Program (*data until 12/30/01)

Figura 2 - Casos notificados de Aids, em indivíduos com categoria de transmissão heterossexual, segundo parceria sexual e sexo, Estado de São Paulo, 1980 a 2001*

Figure 2 -Aids Cases reported, in individuals with heterosexual transmission, according to sexual partnership and gender, State of São Paulo, 1980 to $2001^{*}$

Tabela 5 - Casos notificados de aids, por ano de diagnóstico e categoria de transmissão para menores de 13 anos, no estado de são paulo, período de 1980- 2001(**)

Table 5 - Aids Cases reported, by year of diagnosis and transmission category in individuals under 13 years of age, in the State of São Paulo, 1980-2001

\begin{tabular}{|c|c|c|c|c|c|c|c|c|c|c|c|c|c|c|c|c|c|c|}
\hline \multirow{3}{*}{$\begin{array}{l}\text { Ano de } \\
\text { Diagnóstico }\end{array}$} & \multicolumn{16}{|c|}{ Categoria de Transmissão } & & \\
\hline & \multicolumn{2}{|c|}{ Homo } & \multicolumn{2}{|c|}{ Hetero } & \multicolumn{2}{|c|}{ UDI } & \multicolumn{2}{|c|}{$\mathrm{HSH}+\mathrm{UDI}$} & \multicolumn{2}{|c|}{ Hemof } & \multicolumn{4}{|c|}{ T.sangue $\left.{ }^{*}\right)$ Materno-Inf } & \multicolumn{2}{|c|}{ Invest. } & \multicolumn{2}{|c|}{ TOTAL } \\
\hline & No. & $\%$ & No. & $\%$ & No. & $\%$ & No. & $\%$ & No. & $\%$ & No. & $\%$ & No. & $\%$ & No. & $\%$ & No. & $\%$ \\
\hline 1984 & - & - & - & - & - & - & - & - & 1 & 100.0 & - & - & - & - & - & - & 1 & 100.0 \\
\hline 1985 & - & - & - & - & - & - & - & - & 3 & 75.0 & 1 & 25.0 & - & - & - & - & 4 & 100.0 \\
\hline 1986 & - & - & - & - & - & - & - & - & 6 & 50.0 & 5 & 41.7 & - & - & 1 & 8.3 & 12 & 100.0 \\
\hline 1987 & - & - & - & - & - & - & - & - & 15 & 27.3 & 10 & 18.2 & 26 & 47.3 & 4 & 7.3 & 55 & 100.0 \\
\hline 1988 & 1 & 1.0 & - & - & 2 & 2.0 & - & - & 5 & 5.0 & 17 & 17.0 & 69 & 69.0 & 6 & 6.0 & 100 & 100.0 \\
\hline 1989 & - & - & - & - & 2 & 1.7 & - & - & 10 & 8.3 & 14 & 11.7 & 85 & 70.8 & 9 & 7.5 & 120 & 100.0 \\
\hline 1990 & 1 & 0.6 & - & - & 1 & 0.6 & - & - & 15 & 8.9 & 11 & 6.5 & 128 & 76.2 & 12 & 7.1 & 168 & 100.0 \\
\hline 1991 & - & - & 1 & 0.5 & - & - & - & - & 4 & 1.9 & 10 & 4.7 & 184 & 86.8 & 13 & 6.1 & 212 & 100.0 \\
\hline 1992 & - & - & - & - & - & - & - & - & 5 & 2.0 & 17 & 6.8 & 214 & 85.6 & 14 & 5.6 & 250 & 100.0 \\
\hline 1993 & - & - & - & - & 1 & 0.4 & 1 & 0.4 & 3 & 1.1 & 11 & 4.1 & 230 & 85.8 & 23 & 8.6 & 268 & 100.0 \\
\hline 1994 & - & - & - & - & - & - & - & - & 2 & 0.7 & 8 & 2.7 & 259 & 88.1 & 25 & 8.5 & 294 & 100.0 \\
\hline 1995 & - & - & - & - & - & - & - & - & 2 & 0.6 & 12 & 3.3 & 307 & 85.5 & 38 & 10.6 & 359 & 100.0 \\
\hline 1996 & - & - & - & - & - & - & - & - & 1 & 0.3 & 3 & 0.8 & 335 & 86.3 & 49 & 12.6 & 388 & 100.0 \\
\hline 1997 & - & - & - & - & - & - & - & - & - & - & 1 & 0.3 & 342 & 93.4 & 23 & 6.3 & 366 & 100.0 \\
\hline 1998 & - & - & - & - & - & - & - & - & - & - & 1 & 0.4 & 215 & 83.0 & 43 & 16.6 & 259 & 100.0 \\
\hline 1999 & - & - & - & - & - & - & - & - & 1 & - & 1 & - & 227 & 87.3 & 31 & 11.9 & 260 & 100.0 \\
\hline 2000 & - & - & - & - & - & - & - & - & - & - & 2 & - & 165 & 84.6 & 28 & 14.4 & 195 & 100.0 \\
\hline 2001 & - & - & - & - & - & - & - & - & - & - & 2 & - & 53 & 86.9 & 6 & 9.8 & 61 & 100.0 \\
\hline OTAL & 2 & 0.1 & 1 & 0.0 & 6 & 0.2 & 1 & 0.0 & 73 & 2.2 & 126 & 3.7 & 2839 & 84.2 & 325 & 9.6 & 3372 & 100.0 \\
\hline
\end{tabular}

Fonte: SINAN- Vigilância Epidemiológica - Programa Estadual DST/Aids-SP; HSH - Homens que fazem sexo com homens / UDI - Uso de Drogas Injetáveis; (**) Dados preliminares, sujeitos a revisão mensal, até 31/12/01; $\left(^{*}\right)$ Todos os casos por transfusão de sangue estão sendo reinvestigados de acordo com o algoritmo da CNDST/Aids-MS.

Source: SINAN - Disease Surveillance - STD/Aids State Program - SP; (*) MSM - Men who have sex with men/ IDU - Injecting Drug Users; (**) Preliminary data, subject to monthly revision, until 12/31/01; (\&) All blood transfusion cases are being reinvestigated according to the CNDST/Aids-MS algorithm. 
tância social muito grande, pois denotam a falta de proteção a que muitas crianças estão expostas na nossa sociedade. Os demais casos são conseqüentes à contaminação pela transfusão sangüínea e de hemoderivados que, como para os adultos, vêm diminuindo rapidamente ano a ano.

\section{A mortalidade por aids no ESP}

O pico de mortalidade por aids, no Estado, teve lugar em 1995, ano em que ocorreram 7.739 óbitos, com uma taxa de mortalidade de 22,89 por 100.000 habitantes.

A queda da mortalidade na população masculina aconteceu anteriormente à feminina, além de ocorrer em maior proporção entre os homens do que entre as mulheres, mas a velocidade de decréscimo dos óbitos diminuiu muito nos dois últimos anos, e nas mulheres, particularmente, embora mantendo uma taxa de mortalidade praticamente constante, houve, no Estado de São Paulo, um pequeno acréscimo no número absoluto de óbitos registrados em 2000 (1.241) com relação a 1999 (1.200). Segundo dados do Programa de Aprimoramento das Informações de Mortalidade do Município de São Paulo - PRO-AIM, também em 2001, o número absoluto de óbitos por aids em mulheres, no Município de São Paulo, foi um pouco maior com relação ao ano 2000, $398 \mathrm{e}$ 382 , respectivamente.

Assim, como no número de casos, o declínio da relação masculino/feminino dos óbitos por aids tem demonstrado o avanço da epidemia entre as mulheres.

Na Tabela 6 podemos observar o número de óbitos por aids notificados ao Sistema de Vigilância por ano de ocorrência e por outras fontes de informação - o Sistema de Informações sobre Mortalidade (SIM) e o Sistema de Mortalidade por Aids - SAIDS, produzido pela Fundação Sistema Estadual de Análise de Dados - SEADE, instituição responsável, no Estado de São Paulo, pela produção das estatísticas de óbitos e de nascimentos.

Comparando-se os óbitos por ano de ocorrência dos sistemas de informação, há uma discrepância entre eles. Os números do sistema de VE são expressivamente maiores do que os do SIM até 1990, e são bem menores do que os do SEADE a partir de 1991, com exceção de 1995 e 1998, anos em que o serviço de VE fez um trabalho de resgate manual dos óbitos de aids de todo o Estado, através das Declarações de Óbito (DO) fornecidas pela Fundação SEADE.

Uma possível explicação para isto é que no início da epidemia, por questões sociais e pseudo-éticas, os médicos tivessem maior resistência para colocar o diagnóstico de aids na Declaração de Óbito (DO) e diversos casos evoluíram para óbito por aids, sem que isto constasse da $\mathrm{DO}^{39}$, passando despercebidos pelos sistemas de informação, sendo provável que, num período inicial da epidemia, fosse mais freqüente o registro dos óbitos de pacientes já notificados. A partir de 1991, com o grande número de óbitos ocorridos e a banalização do diagnóstico, mais casos foram sendo relatados oficialmente nas DO e, antagonicamente, esta mesma banalização talvez tenha levado a uma diminuição da sensibilidade dos profissionais para a notificação dos casos de aids, aumentando, portanto, o número de casos conhecidos apenas através do sistema de mortalidade. Além disto, desde 1990, o PRO-AIM e a Fundação SEADE têm trabalhado com as DO suspeitas de aids e encaminhado para investigação.

Comparando os coeficientes de mortalidade por sexo (Tabela 7) vê-se que o pico da mortalidade ocorreu em 1994/1995 para os homens e em 1995/1996 para as mulheres, declinando depois mais intensamente na população masculina do que na feminina. A razão masculino/feminino dos óbitos é semelhante à dos casos, atinge seu pico em $1986(38,6 / 1)$ e diminui gradativamente até chegar a 2,36 no ano 2000.

Na faixa etária do adulto jovem, segundo os dados do PRO-AIM e da Fundação SEADE, a aids figura entre as principais causas de mortalidade, tanto no Município quanto no Estado de São Paulo, tendo sido a principal causa de mortalidade das mulheres em idade fértil de 1991 a 1998. Em 1999 e 2000, a aids foi 
Tabela 6 - Óbitos de aids, coeficiente de mortalidade* $(\mathrm{cm})$ por 100.000 habitantes, segundo ano de diagnóstico, estado de são paulo, 1980 a 2000 (**) $^{* *}$

Table 6 - Deaths due to Aids, mortality coefficient* (MC) by 100,000 inhabitants, according to year of diagnosis, State of São Paulo, 1980 to $\left.2000{ }^{* *}\right)$

\begin{tabular}{ccccc}
\hline $\begin{array}{c}\text { Ano de } \\
\text { Diagnóstico }\end{array}$ & $\begin{array}{c}\text { Óbitos* } \\
\text { (SIM/SEADE) }\end{array}$ & CM & $\begin{array}{c}\text { Óbitos } \\
\text { (SINAN) }\end{array}$ & CM \\
\hline 1980 & 1 & - & - & - \\
1981 & - & - & 1 & - \\
1982 & 0 & 0.00 & 2 & 0.01 \\
1983 & 1 & 0.00 & 16 & 0.06 \\
1984 & 0 & 0.00 & 50 & 0.18 \\
1985 & 74 & 0.27 & 173 & 0.62 \\
1986 & 198 & 0.70 & 297 & 1.05 \\
1987 & 437 & 1.51 & 705 & 2.44 \\
1988 & 1075 & 3.64 & 1379 & 4.67 \\
1989 & 1665 & 5.52 & 2188 & 7.26 \\
1990 & 3098 & 10.06 & 3179 & 10.33 \\
1991 & 4218 & 13.42 & 4103 & 13.05 \\
1992 & 5021 & 15.68 & 4761 & 14.87 \\
1993 & 6433 & 19.78 & 5676 & 17.45 \\
1994 & 7091 & 21.46 & 6273 & 18.98 \\
1995 & 7739 & 22.95 & 7528 & 22.33 \\
1996 & 7269 & 21.33 & 6763 & 19.85 \\
1997 & 5536 & 16.01 & 4242 & 12.27 \\
1998 & 4591 & 13.07 & 4647 & 13.23 \\
1999 & 4257 & 11.96 & 3562 & 10.01 \\
2000 & 4181 & - & 3568 & - \\
\hline Total & 59434 & & 59113 & \\
\hline
\end{tabular}

Fonte: SINAN-Vigilância Epidemiológica - Programa Estadual DST/Aids-SP; *óbitos: SIM-DATASUS (1980 a 1989) e SEADE (1990 a 2000); (**) Dados preliminares, sujeitos a revisão mensal, até 31/12/01; (**) Utilizada projeção populacional da Fundação SEADE; não incluídos 9 óbitos sem data.

Source: SINAN - Disease Surveillance - STD/Aids State Program - SP; *deaths: SIM-DATASUS (1980 to 1989) and SEADE (1990 to 2000); (**) Preliminary data, subject to monthly revision, until 12/31/01; (***)Fundação SEADE's populational projection was used 9 deaths without a date were not included.

a segunda causa de morte para as mulheres entre 15 e 49 anos, no Estado de São Paulo, tendo surgido, como primeira causa, as Doenças Cerebro-Vasculares. Para os homens, a aids oscila, de 1991 até o ano 2000, entre a segunda e a terceira causa, estando, para estes, em primeiro lugar os homicídios e os acidentes de trânsito. No ano de 1998, a aids foi a oitava causa de morte, em todas as idades no ESP, sendo a sexta para os homens e a décima para as mulheres.

O comportamento apresentado pela mortalidade por aids, segundo a idade, é diferente nas populações masculina e feminina. Entre 1988 e 1997, o pico de idade para a mortalidade por aids foi entre 30-34 anos na população masculina e, para as mulheres, entre 25-29 anos $^{40}$.

Analisando a mortalidade por aids no ESP, de 1990 até o ano 2000, Waldvogel (2002) declara: "Para os homens, observa-se uma redução nas taxas de mortalidade por Aids na população com até 30 anos de idade, quando se comparam os dados de 1990 e 2000. A partir deste corte etário, as taxas aproximam-se bastante .... que difere em relação às mulheres é que, para esta parcela da população, a desaceleração da incidência e da mortalidade por aids foi menor, de forma que a curva descrita pelas taxas de mortalidade por idade, em 2000, ainda não está próxima da correspondente a 1990. Note-se 
Tabela 7 - Coeficiente de mortalidade por aids (por 100.000 habitantes) e razão proporcional de óbitos masculinos e femininos, segundo sexo e ano de ocorrência, estado de são paulo, 1980 a $2000(* *)$

Table 7- Aids mortality coefficients (by 100,000 inhabitants) and proportional male and female death ratio, according to gender and year of occurrence State of São Paulo, 1980 to $\left.2000^{* * *}\right)$

\begin{tabular}{|c|c|c|c|c|c|}
\hline \multirow{4}{*}{$\begin{array}{c}\text { Ano de } \\
\text { Óbito }\end{array}$} & \multicolumn{4}{|c|}{ Sexo } & \multirow{4}{*}{$\begin{array}{c}\text { Razão } \\
\text { OB. M/F } \\
\text { SIM/SEADE* }\end{array}$} \\
\hline & \multicolumn{2}{|c|}{ Masculino } & \multicolumn{2}{|c|}{ Feminino } & \\
\hline & CM & & CM & & \\
\hline & SIM/SEADE* & V.E. & SIM/SEADE* & V.E. & \\
\hline 1980 & - & - & - & - & - \\
\hline 1981 & - & 0.01 & - & - & - \\
\hline 1982 & - & 0.02 & - & - & - \\
\hline 1983 & 0.01 & 0.12 & - & - & - \\
\hline 1984 & - & 0.37 & - & - & - \\
\hline 1985 & 0.51 & 1.23 & 0.03 & 0.03 & 17.5 \\
\hline 1986 & 1.37 & 2.05 & 0.04 & 0.06 & 38.6 \\
\hline 1987 & 2.83 & 4.46 & 0.21 & 0.45 & 13.10 \\
\hline 1988 & 6.41 & 8.23 & 0.92 & 1.18 & 6.85 \\
\hline 1989 & 9.58 & 12.77 & 1.54 & 1.85 & 6.12 \\
\hline 1990 & 17.30 & 18.03 & 2.97 & 2.78 & 5.73 \\
\hline 1991 & 22.50 & 22.00 & 4.54 & 4.30 & 4.82 \\
\hline 1992 & 26.02 & 24.94 & 5.60 & 5.05 & 4.54 \\
\hline 1993 & 32.20 & 28.59 & 7.70 & 6.62 & 4.10 \\
\hline 1994 & 34.45 & 30.66 & 8.85 & 7.66 & 3.77 \\
\hline 1995 & 35.44 & 34.60 & 11.08 & 10.66 & 3.09 \\
\hline 1996 & 32.09 & 29.92 & 10.95 & 10.12 & 2.83 \\
\hline 1997 & 23.35 & 17.69 & 8.86 & 6.98 & 2.56 \\
\hline 1998 & 18.80 & 19.10 & 7.50 & 7.52 & 2.43 \\
\hline 1999 & 17.44 & 14.70 & 6.65 & 5.45 & 2.55 \\
\hline 2000 & 16.26 & 13.82 & 6.59 & 5.86 & 2.36 \\
\hline
\end{tabular}

Fonte: SINAN-Vigilância Epidemiológica - Programa Estadual DST/Aids-SP; *óbitos: SIM-DATASUS (1980 a 1989) e SEADE (1990 a 2000); (**) Dados preliminares, sujeitos a revisão mensal, até 31/12/01; (**) Utilizada projeção populacional da Fundação SEADE. Source: SINAN - Disease Surveillance - STD/Aids State Program - SP; *deaths: SIM-DATASUS (1980 to 1989) and SEADE (1990 to 2000); (**) Preliminary data, subject to monthly revision, until 12/31/01; (***)Fundação SEADE's populational projection.was used

também que, para as mulheres, houve uma defasagem nas idades mais atingidas pela Aids: em 1990, a faixa etária mais afetada situa-se entre 20 e 29 anos; em 2000, ela corresponde às idades entre 30 e 39 anos, semelhante ao padrão verificado na população masculina. Por outro lado, os homens com mais de 35 anos registram um padrão distinto do descrito até o momento. Os casos fatais de aids, observados em tal parcela da população, apresentam três movimentos: um primeiro aumento até atingir um máximo na metade da década, uma diminuição até 1998/1999 e um novo aumento entre 1999/2000 ... As mulheres com mais de 35 anos registram padrão semelhante ao ve- rificado pelos homens desta idade, com três movimentos na curva descrita pelos casos fatais de aids." ${ }^{41}$

Apesar do quadro otimista com relação à evolução da mortalidade da aids em São Paulo, a diminuição da velocidade da queda dos óbitos e o pequeno aumento no número absoluto de casos em mulheres e das taxas de mortalidade em indivíduos maiores de 35 anos, são fatos que devem colocar em alerta os programas de prevenção e controle de DST/AIDS, buscando entender até onde vai o impacto das terapias anti-retrovirais hoje existentes e que outros fatores podem influenciar a dinâmica da mortalidade por aids em nosso meio. 


\section{Juvenilização ou envelhecimento?}

A aids aparece em todas as faixas etárias, sendo mais prevalente no adulto jovem. Com o objetivo de verificar a distribuição por idade dos casos de aids, foram avaliados 101.503 casos diagnosticados de 1980 a 1998 e calculados os coeficientes de incidência segundo idade, sexo e principais categorias de exposição.

O que se observa é uma proporção mais ou menos constante de casos em cada faixa etária ao longo dos anos, em ambos os gêneros. No masculino, a faixa etária dos 30-39 anos mantém-se como a que apresenta maiores coeficientes de incidência em todo o período estudado, seguida pela faixa de 20 29 anos. Observa-se, nesta última faixa etária, uma tendência decrescente a partir de 1995 (Figura 3), relacionada à queda dos casos entre usuários de drogas injetáveis, que mostrou maior impacto entre os homens jovens. Há, na verdade, um ligeiro aumento das fai- xas etárias mais avançadas, de 40-49 e 50-59, que, se mantido, pode indicar um leve "envelhecimento" da epidemia.

Nas mulheres, ao contrário de juvenilização, há um nítido crescimento dos coeficientes de incidência na faixa etária de 30-39 (22,42 casos por 100.000 mulheres em 1994 para 46,99 em 1998) (Figura 4), que vem em segundo lugar até 1994 e, depois deste ano, passa a ser a mais prevalente entre os casos de aids femininos. As demais faixas apresentam-se na mesma proporção desde o início da epidemia, com tendência de crescimento dos coeficientes nas faixas dos 20 aos 49 anos. O coeficiente, entre as mulheres de 40 a 49 anos, apresentou aumento importante $(4,40$ em 1990 e 23,63 em 1998).

Entre as mulheres heterossexuais nota-se uma diminuição na proporção de casos entre 20 a 29 anos após 1993 e aumento entre 30 a 39 anos. Para os homens heterossexuais, a maior proporção de casos permaneceu entre os 30 e 39 anos em todo o período.

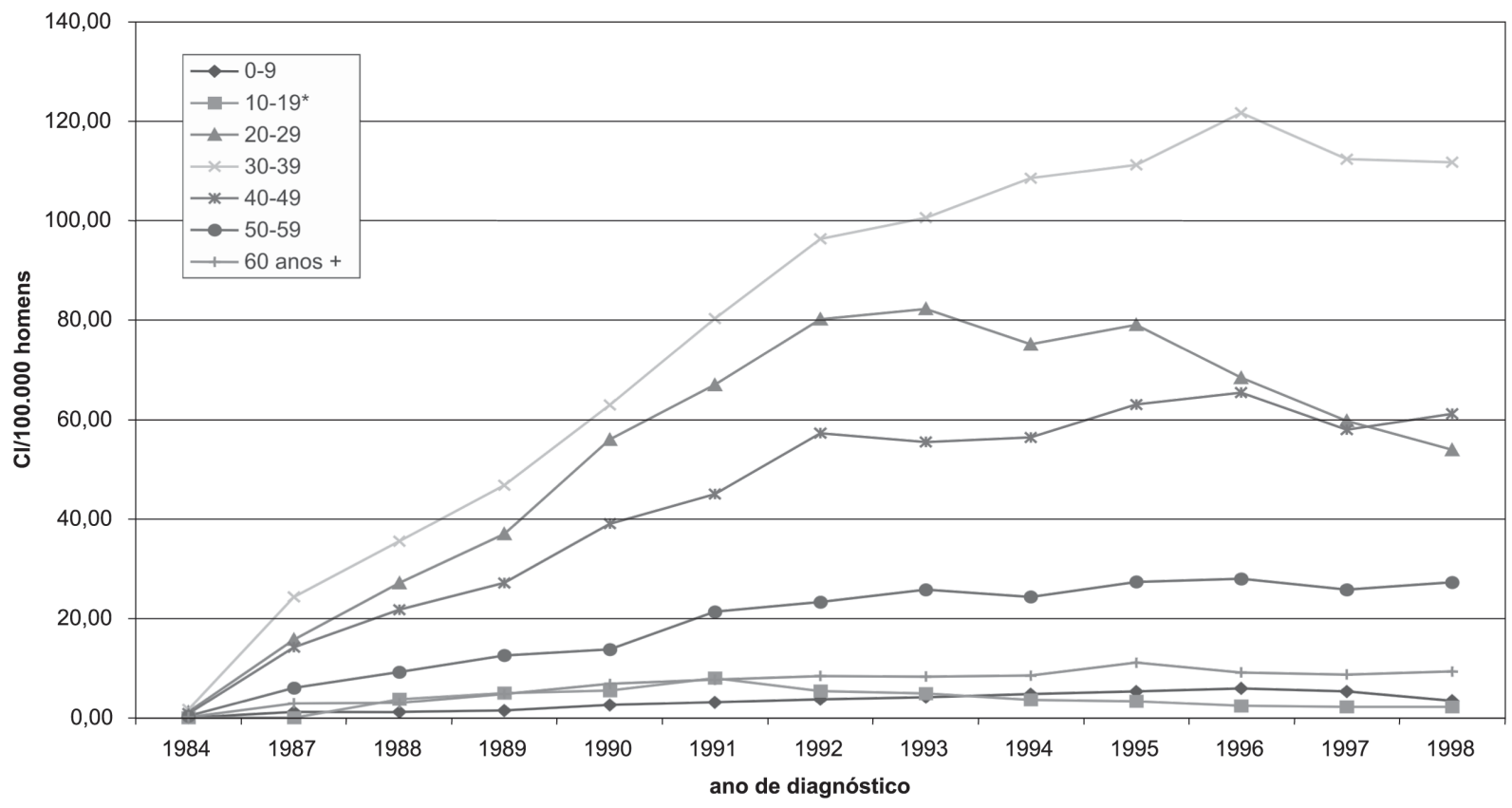

Fonte:Vigilância Epidemiológica -Programa Estadual DST/Aids e *SEADE; ***dados até 31/05/2001.

Source: Disease Surveillance - São Paulo State STD/Aids Program and *SEADE; ***data until 05/31/2001.

Figura 3 - Aids Coeficiente de Incidência (Cl) no sexo masculino (por 100.000 homens*) segundo faixa etária e ano de diagnóstico, Estado de São Paulo, 1984 a 1998(***)

Figure 3 - Aids Incidence Coefficients (IC) for men (by 100,000 men*) according to age group and year of diagnosis, State of São Paulo, 1984 to 1998 (**) $^{* *}$ 


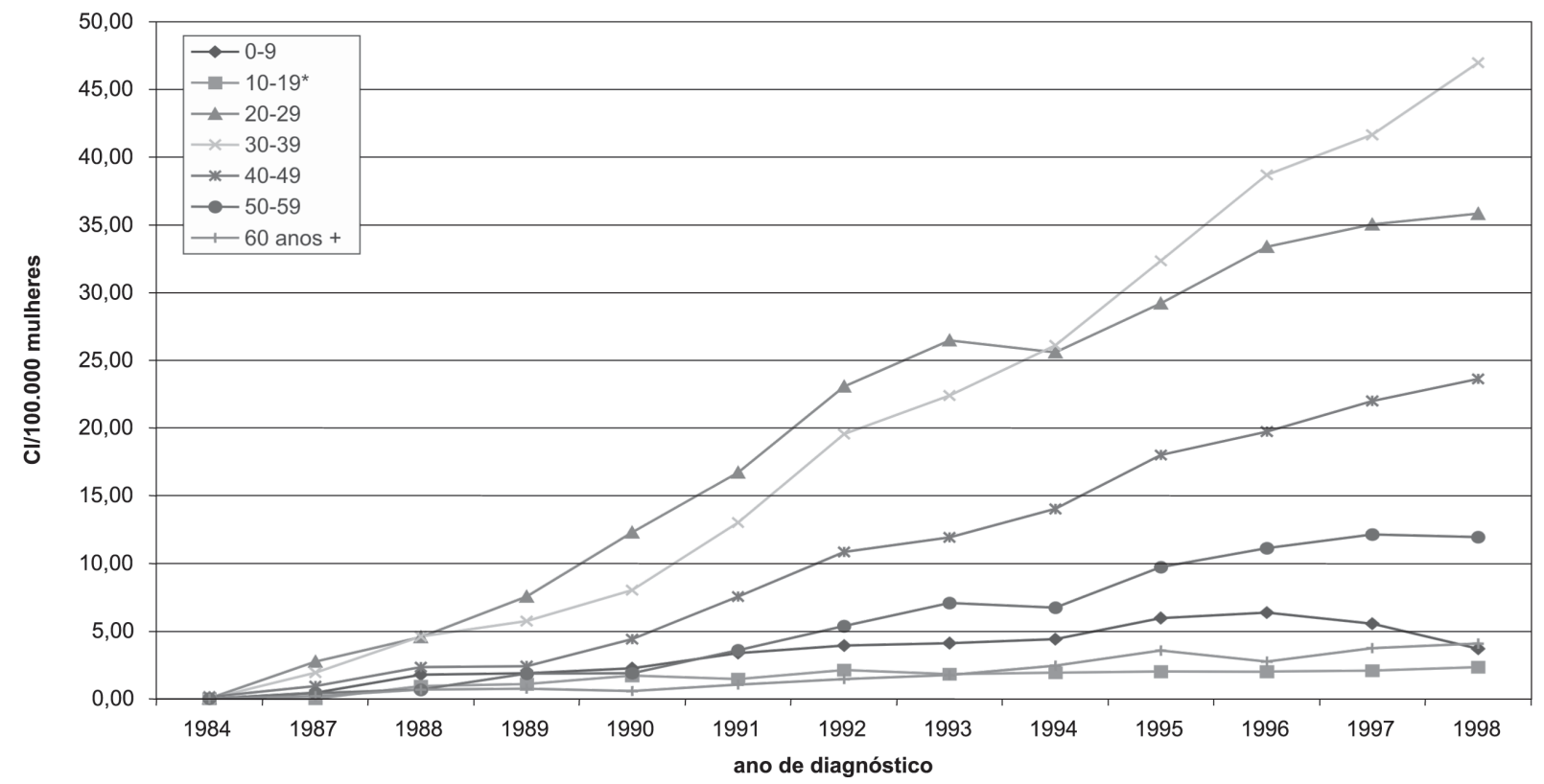

Fonte: Vigilância Epidemiológica -Programa Estadual DST/Aids e *SEADE; ***dados até 31/05/2001.

Source: Disease Surveillance - São Paulo State STD/Aids Program and *SEADE; ${ }^{* *}$ data until 05/31/2001.

Figura 4 - Aids - Coeficiente de Incidência (Cl) no sexo feminino (por 100.000 mulheres*) segundo faixa etária e ano de diagnóstico, Estado de São Paulo, 1984 a 1998(***)

Figure 4 - Aids - Incidence Coefficients (IC) for women (by 100,000 women*) according to age group and year of diagnosis

A proporção de casos em homens usuários de drogas injetáveis (UDI) de 20 a 29 anos foi predominante até 1995 (48,06\%), diminuindo rapidamente após 1992. O grupo etário de 30 a 39 anos passa a ser mais freqüente após 1996. A proporção de mulheres de 20 a 29 anos com categoria de exposição de UDI também foi a mais freqüente até 1996, sendo, nesse ano, ultrapassada pelas mulheres mais velhas (de 30 a 39 anos).

Entre os casos de homens que fazem sexo com homens (HSH), a idade predominante durante todo o período foi de 30 a 39 anos, e foi observada tendência de queda entre os casos diagnosticados de 20 a 29 anos.

Sendo assim, não se observa no Estado de São Paulo, como um todo, o fenômeno da juvenilização da epidemia de aids, e esta hipótese não pode ser confirmada pelos casos notificados no Estado de São Paulo, a menos que haja diferenças mais sutis que possam ser percebidas em determinadas regiões geográficas ou, ainda , através da vigilância e notificação de indivíduos infecta- dos pelo HIV que ainda não manifestaram sintomas da aids.

A análise dos dados de mortalidade por aids, no Estado, descrita anteriormente, também aponta um deslocamento dos óbitos para faixas etárias mais altas, contrariando a hipótese de juvenilização e levando a crer que está havendo um envelhecimento das pessoas atingidas pela aids.

\section{Pauperização}

A escolaridade e a ocupação têm sido usadas como marcadores das condições sócio-econômicas dos pacientes com aids.

A aids aparece em todas as camadas sociais, sendo que ao longo dos anos tem havido um aumento do número de casos entre pacientes com escolaridade mais baixa e ocupações menos qualificadas. Um fato interessante de se observar é que, embora todas as categorias de transmissão apareçam entre as diversas camadas sociais, o uso de drogas injetáveis é proporcionalmente mais 
importante entre os pacientes com baixa escolaridade e ocupações menos qualificadas, e a transmissão sexual é proporcionalmente mais importante nos indivíduos de escolaridade mais alta, em ambos os se$\operatorname{xos}^{14,42}$.

Observa-se nas Tabelas 8 e 9, a proporção de casos notificados de aids segundo o grau de instrução, para homens e mulheres.

Os casos masculinos têm uma proporção muito maior de indivíduos de nível superior do que as mulheres, especialmente nos primeiros anos da epidemia. Isto se deve ao fato de que, neste período, a categoria de exposição mais comum era a homossexual e que estes indivíduos apresentavam, em média, escolaridade muito maior do que a população em geral ${ }^{13,44}$.

Observa-se, ainda na Tabela 8, que, a partir do início dos anos 90, a proporção de pessoas com primeiro grau completo e incompleto cresce entre os casos masculinos notificados, período este que corresponde à explosão dos casos transmitidos pelo uso de drogas injetáveis, que está sabidamente relacionada com uma parcela menos privilegiada, do ponto de vista sócio-econômico, da população $0^{9,14,44}$.

Esta diminuição do grau de instrução dos pacientes com aids tem sido largamente citada como indicador de pauperização $0^{9,20,42}$ da epidemia.

Tabela 8 - Casos notificados de aids em homens maiores de 12 anos, segundo escolaridade* e ano de diagnóstico, estado de são paulo, 1980 - 2001 (**). $^{*}$.

Table 8 - Aids Cases reported in men above 12 years of age, according to schooling* and year of diagnosis, State of São Paulo, 1980 $-2001(* *)$

\begin{tabular}{|c|c|c|c|c|c|c|c|c|c|c|}
\hline \multirow{3}{*}{$\begin{array}{c}\text { Ano de } \\
\text { Diagnóstico }\end{array}$} & \multicolumn{8}{|c|}{ Grau de Escolaridade } & & \\
\hline & \multicolumn{2}{|c|}{ Analfabeto } & \multicolumn{2}{|c|}{$1{ }^{\circ} \mathrm{Grau}$} & \multicolumn{2}{|c|}{$2^{\circ} \mathrm{Grau}$} & \multicolumn{2}{|c|}{ Superior } & \multicolumn{2}{|c|}{ Total } \\
\hline & No. & $\%$ & No. & $\%$ & No. & $\%$ & No. & $\%$ & No. & $\%$ \\
\hline 1980 & - & - & - & - & 1 & 100.0 & - & - & 1 & 100.0 \\
\hline 1982 & - & - & 1 & 33.3 & 1 & 33.3 & 1 & 33.3 & 3 & 100.0 \\
\hline 1983 & - & - & 4 & 40.0 & 2 & 20.0 & 5 & 50.0 & 10 & 100.0 \\
\hline 1984 & - & - & 18 & 45.0 & 8 & 20.0 & 16 & 40.0 & 40 & 100.0 \\
\hline 1985 & 2 & 1.8 & 40 & 35.1 & 20 & 17.5 & 52 & 45.6 & 114 & 100.0 \\
\hline 1986 & 2 & 0.9 & 89 & 40.3 & 48 & 21.7 & 82 & 37.1 & 221 & 100.0 \\
\hline 1987 & 17 & 2.5 & 338 & 49.3 & 150 & 21.9 & 181 & 26.4 & 686 & 100.0 \\
\hline 1988 & 22 & 1.6 & 728 & 53.6 & 302 & 22.2 & 306 & 22.5 & 1358 & 100.0 \\
\hline 1989 & 49 & 2.4 & 1177 & 58.7 & 416 & 20.8 & 362 & 18.1 & 2004 & 100.0 \\
\hline 1990 & 46 & 1.6 & 1727 & 59.2 & 636 & 21.8 & 507 & 17.4 & 2916 & 100.0 \\
\hline 1991 & 57 & 1.6 & 2247 & 61.5 & 812 & 22.2 & 539 & 14.7 & 3655 & 100.0 \\
\hline 1992 & 104 & 2.2 & 3012 & 63.6 & 992 & 21.0 & 626 & 13.2 & 4734 & 100.0 \\
\hline 1993 & 154 & 3.1 & 3190 & 64.0 & 1075 & 21.6 & 564 & 11.3 & 4983 & 100.0 \\
\hline 1994 & 143 & 2.8 & 3361 & 66.1 & 1053 & 20.7 & 527 & 10.4 & 5084 & 100.0 \\
\hline 1995 & 168 & 3.1 & 3652 & 67.2 & 1109 & 20.4 & 507 & 9.3 & 5436 & 100.0 \\
\hline 1996 & 196 & 3.6 & 3734 & 68.6 & 1026 & 18.8 & 488 & 9.0 & 5444 & 100.0 \\
\hline 1997 & 195 & 3.7 & 3633 & 69.0 & 1012 & 19.2 & 423 & 8.0 & 5263 & 100.0 \\
\hline 1998 & 165 & 3.0 & 3958 & 71.4 & 944 & 17.0 & 473 & 8.5 & 5540 & 100.0 \\
\hline 1999 & 136 & 2.9 & 3374 & 72.5 & 783 & 16.8 & 360 & 7.7 & 4653 & 100.0 \\
\hline 2000 & 120 & 3.1 & 2759 & 71.2 & 709 & 18.3 & 285 & 7.4 & 3873 & 100.0 \\
\hline 2001 & 53 & 3.3 & 1108 & 68.9 & 329 & 20.5 & 117 & 7.3 & 1607 & 100.0 \\
\hline Total & 1629 & 2.8 & 38150 & 66.3 & 11428 & 19.9 & 6304 & 11.0 & 57511 & 100.0 \\
\hline
\end{tabular}

Fonte: Vigilância Epidemiológica - Programa Estadual DST/Aids; (**) Dados preliminares, sujeitos a revisão mensal, até 31/12/01; ** Não incluídos 19.410 homens com escolaridade sem informação.

Source: SINAN - Disease Surveillance -STD/AIDS State Program - SP STD/AIDS State Program - SP; Preliminary data, subject to monthly revision until 12/30/01; Does not include 19,410 men without information on schooling. 
Tabela 9 - Casos notificados de aids mulheres maiores de 12 anos, segundo escolaridade* e ano de diagnóstico, estado de são paulo, 1980 - 2001(**).

Table 9 -Aids Cases reported in women above 12 years of age, according to schooling* and year of diagnosis, State of São Paulo, $1980-2001(* *)$

\begin{tabular}{|c|c|c|c|c|c|c|c|c|c|c|}
\hline \multirow{3}{*}{$\begin{array}{c}\text { Ano de } \\
\text { Diagnóstico }\end{array}$} & \multicolumn{8}{|c|}{ Grau de Escolaridade } & & \\
\hline & \multicolumn{2}{|c|}{ Analfabeto } & \multicolumn{2}{|c|}{$1^{\circ} \mathrm{Grau}$} & \multicolumn{2}{|c|}{$2^{\circ} \mathrm{Grau}$} & \multicolumn{2}{|c|}{ Superior } & \multicolumn{2}{|c|}{ Total } \\
\hline & No. & $\%$ & No. & $\%$ & No. & $\%$ & No. & $\%$ & No. & $\%$ \\
\hline 1980 & - & - & - & - & - & - & - & - & - & - \\
\hline 1982 & - & - & - & - & - & - & - & - & - & - \\
\hline 1983 & - & - & 1 & 100.0 & - & - & - & - & 1 & 100.0 \\
\hline 1984 & - & - & 1 & 50.0 & 1 & 50.0 & - & - & 2 & 100.0 \\
\hline 1985 & - & - & 7 & 87.5 & 2 & 25.0 & - & - & 8 & 100.0 \\
\hline 1986 & - & - & 4 & 80.0 & 3 & 60.0 & 1 & 20.0 & 5 & 100.0 \\
\hline 1987 & 6 & 7.4 & 58 & 71.6 & 12 & 14.8 & 5 & 6.2 & 81 & 100.0 \\
\hline 1988 & 7 & 3.4 & 156 & 76.1 & 31 & 15.1 & 11 & 5.4 & 205 & 100.0 \\
\hline 1989 & 23 & 7.4 & 223 & 72.2 & 45 & 14.6 & 18 & 5.8 & 309 & 100.0 \\
\hline 1990 & 16 & 3.2 & 357 & 72.4 & 91 & 18.5 & 29 & 5.9 & 493 & 100.0 \\
\hline 1991 & 35 & 4.9 & 539 & 75.2 & 109 & 15.2 & 34 & 4.7 & 717 & 100.0 \\
\hline 1992 & 53 & 4.8 & 782 & 70.5 & 206 & 18.6 & 69 & 6.2 & 1110 & 100.0 \\
\hline 1993 & 78 & 5.7 & 984 & 72.4 & 241 & 17.7 & 56 & 4.1 & 1359 & 100.0 \\
\hline 1994 & 92 & 6.2 & 1094 & 73.6 & 236 & 15.9 & 64 & 4.3 & 1486 & 100.0 \\
\hline 1995 & 97 & 5.2 & 1350 & 72.6 & 337 & 18.1 & 76 & 4.1 & 1860 & 100.0 \\
\hline 1996 & 114 & 5.4 & 1541 & 72.8 & 374 & 17.7 & 87 & 4.1 & 2116 & 100.0 \\
\hline 1997 & 150 & 6.0 & 1858 & 74.4 & 394 & 15.8 & 95 & 3.8 & 2497 & 100.0 \\
\hline 1998 & 141 & 5.0 & 2160 & 77.1 & 394 & 14.1 & 105 & 3.8 & 2800 & 100.0 \\
\hline 1999 & 94 & 3.9 & 1872 & 77.5 & 364 & 15.1 & 86 & 3.6 & 2416 & 100.0 \\
\hline 2000 & 100 & 4.9 & 1549 & 75.8 & 317 & 15.5 & 78 & 3.8 & 2044 & 100.0 \\
\hline 2001 & 46 & 5.4 & 646 & 75.2 & 129 & 15.0 & 38 & 4.4 & 859 & 100.0 \\
\hline Total & 1052 & 5.2 & 15182 & 74.5 & 3286 & 16.1 & 852 & 4.2 & 20372 & 100.0 \\
\hline
\end{tabular}

Fonte: SINAN- Vigilância Epidemiológica - Programa Estadual DST/Aids-SP; (**) Dados preliminares, sujeitos a revisão mensal, até 31/12/01.; Não incluídos 5.907 mulheres com escolaridade sem informação.

Source: SINAN - Disease Surveillance - STD/AIDS State Program - SP; Preliminary data, subject to monthly revision until 12/30/01; Does not include 5,907 women without information on schooling

Nas mulheres, entretanto, não há evidência de deslocamento do grau de instrução dos casos ao longo dos anos pois, desde os primeiros anos, a maioria dos casos femininos está entre aquelas com escolaridade menor ou igual ao primeiro grau, e a proporção de analfabetas é maior do que para os homens. A proporção de mulheres com escolaridade superior fica em torno de $4 \%$, mantendo-se relativamente constante no decorrer dos anos.

Assim a pauperização da epidemia é um fato que se observa claramente na epidemia masculina, mas não na feminina. A epidemia de aids entre as mulheres está pauperizada desde o seu início, mantendo entretan- to uma porcentagem de casos entre as mulheres universitárias mais ou menos constante ao longo dos anos.

É importante ressaltar este fato, pois o discurso da pauperização da epidemia dá às mulheres com maior escolaridade e melhor nível sócio-econômico uma falsa sensação de "proteção" e distanciamento da doença. Erro semelhante foi cometido no início da epidemia, quando o alarde sobre o fato de que a maioria dos casos ocorria em homens homossexuais, levou os demais segmentos da sociedade a se acreditarem "fora do risco". Esta crença foi, provavelmente, um dos fatores facilitadores da heterossexualização da epidemia. 


\section{Feminização e heterossexualização}

A epidemia de aids no Estado de São Paulo cresceu rapidamente, atingindo seu pico máximo de incidência entre 1995 e 1996 para os homens e, depois disto, começou a declinar. Nas mulheres apresentou até 1998 uma tendência ascendente.

Muito tem se discutido a respeito da diminuição da incidência de casos, o que na verdade se verifica mais claramente entre os homens. Entretanto, mesmo entre estes, que referem à prática heterossexual como categoria de exposição, há uma tendência de crescimento até 1997, acompanhando a tendência das mulheres.

Ainda que se acredite que o número de casos entre homens heterossexuais esteja superestimado, e que uma parcela destes homens sejam bissexuais não declarados, supõe-se que isto não aconteça na maioria dos casos. Esta tendência representa, de fato, o modo de ocorrência da epidemia na população heterossexual masculina, até porque os homens homo e bissexuais têm apresentado um padrão de aumento e diminuição de casos semelhante ao longo do tempo, inclusive com relação a um pequeno aumento do número de casos em 1998, contrariando a tendência de queda, ano a ano, dos indivíduos que relatam estas categorias de exposição.

$\mathrm{O}$ crescimento dos casos entre homens heterossexuais, junto ao marcante predomínio desta forma de transmissão na população feminina, corrobora a hipótese de heterossexualização da epidemia.

Há que se considerar, também, que a imprensa e mesmo alguns técnicos da saúde não facilitaram a percepção de risco da população heterossexual, particularmente a masculina, e incutiram a idéia de que "homem não pega aids de mulher" ou que "é muito difícil um homem ser infectado pelo HIV por uma mulher".

Vários estudos têm investigado a probabilidade de transmissão do HIV nas relações heterossexuais ${ }^{45,46}$, e seus resultados indicam quase sempre que a possibilidade de transmissão homem-mulher é maior do que a transmissão mulher-homem. O que tem variado muito nestes estudos é o quanto esta possibilidade é maior. Um estudo Europeu envolvendo 525 pares discordantes, 378 casais onde o homem era o caso índice e 148 casais onde o caso índice era a mulher, concluiu que a infectividade era maior do homem para a mulher, mas não significativamente $^{47}$.

A feminização da epidemia é fato consumado. O decréscimo da razão masculino/ feminino de casos e de óbitos e o fato da aids figurar entre as principais causas de mortalidade nas mulheres em idade fértil demonstram, inequivocamente, a magnitude com que a aids tem atingido nossa população feminina.

Como já foi observado, o uso de drogas injetáveis foi um fator fundamental na feminização da epidemia no Estado de São Paulo, e este mesmo fato ocorreu em outros locais do mundo, como na Europa ${ }^{7}$ e nos EUA ${ }^{6}$.

Um aspecto essencial na epidemia de aids é que, ao contrário da fantasia da maioria da população e mesmo dos trabalhadores da saúde, há muito tempo ela já não está restrita a "grupos de risco" específicos e isto é mais facilmente observado na população feminina ${ }^{48-50}$. Uma parcela cada vez mais expressiva das pacientes notificadas de aids tem parceria sexual única no momento do diagnóstico e um número de parceiros sexuais na vida que não difere muito do relatado pela maioria da população ${ }^{51}$.

Considerando o perfil dos casos notificados de aids no Estado de São Paulo, podemos, facilmente destacar alguns fatores que indicam uma maior vulnerabilidade das mulheres: o fato de elas terem menor escolaridade, entendido como condição sócioeconômica menos privilegiada, e uma maior proporção, entre os homens heterossexuais, de multiplicidade de parceiras e, ainda, uma maior proporção de mulheres que apresentam como categoria de exposição para o HIV parceiros com múltiplas parceiras sexuais, reiterando o fato de que não podemos subestimar a forte associação das relações de gênero nas sociedades com maior 
vulnerabilidade das mulheres à infecção pelo $\mathrm{HIV}^{52}$.

Há que se considerar, ainda, que o aumento do número de casos em mulheres traz, como conseqüência, o aumento no número de crianças com aids pela transmissão materno-infantil e traz à tona o problema da orfandade ${ }^{53}$.

\section{Perspectivas da vigilância epidemiológica do HIV/aids}

O atual sistema de vigilância epidemiológica de aids é baseado principalmente na notificação de casos que preenchem os critérios de definição de aids. Esse sistema tem sido utilizado como principal fonte de informação para observação das tendências da epidemia e para o planejamento das atividades de prevenção e assistência, assim como para a divulgação da doença para a população geral.

A vigilância epidemiológica de aids reflete uma situação de vários anos após a infecção ter acontecido, e este intervalo de tempo tende a aumentar em virtude de diversos fatores, tais como a introdução dos antiretrovirais, incorporação de profilaxia e tratamento de doenças oportunistas, e facilidade de acesso aos serviços pela descentralização, levando ao aumento do tempo que os indivíduos infectados pelo HIV permanecem sem sintomas, ou seja, esses casos entrarão cada vez mais tardiamente no sistema de informação, fazendo com que as informações do sistema atual fiquem cada vez mais distantes da real magnitude da infecção pelo HIV.

Apesar de reconhecer sua relevância e utilidade, algumas desvantagens com relação aos sistemas de vigilância de aids devem ser consideradas: nestes, a mensuração dos comportamentos de risco que servem para dar o sinal de alarme quanto à disseminação do HIV, acontece tardiamente, e por isso é difícil explicar as modificações na magnitude da infecção pelo HIV, especialmente nos países onde se dispõe de tratamento com os sistemas atuais; rotineiramente, suas informações não são cruzadas com as de outras fontes, mas apenas em atividades ou momentos específicos; e os recursos da vigilância muitas vezes se destinam à população geral, cujo risco de infecção é baixo, em vez de às populações sob maior risco.

Diante deste quadro, outras estratégias têm sido implementadas para se avaliar as tendências da infecção pelo HIV e para subsidiar novas atividades de prevenção e controle.

\section{Sistema de Informação de Gestantes HIV positivas e Crianças Expostas}

Com a possibilidade concreta de redução da transmissão materno-infantil do HIV, através de uma intervenção eficaz dos serviços de saúde, com o uso do AZT na gestação, durante o trabalho de parto e nas primeiras semanas de vida das crianças filhas de mulheres HIV positivas, tornou-se fundamental o oferecimento do teste anti-HIV às gestantes e a sistematização das informações relativas a esta forma de transmissão do HIV.

O Governo do Estado de São Paulo, através da Lei 10.449 de 20 de dezembro de 1999, instituiu a obrigatoriedade do oferecimento às gestantes do teste para detecção de anticorpos anti-HIV e da Sífilis em todo prénatal realizado pelos serviços públicos e privados no Estado de São Paulo.

O Ministério da Saúde, através da Portaria N. ${ }^{\circ} 0993$, de 4 de setembro de 2000, tornou compulsória a notificação de gestantes HIV positivas e crianças expostas ao HIV (e das Hepatites Virais) em todo o território nacional. A partir dessa data foi iniciado o processo de implantação do instrumento de notificação e investigação de casos dessa população em todo o país.

A implantação do sistema de vigilância do HIV na gestação possibilita medidas oportunas para a redução da transmissão da infecção pelo HIV para o concepto, além do fato de a informação da prevalência da infecção entre as gestantes ser um importante indicador da infecção entre as mulheres, possibilitando a detecção precoce de mudanças no perfil epidemiológico da infecção, 
e conseqüente planejamento das ações para o controle da doença.

\section{Notificação de HIV Positivos Assintomáticos.}

O Estado de São Paulo, tem demonstrado desde o início da década de 90, a intenção de trabalhar com a notificação dos portadores assintomáticos do HIV, entendendo que, dado o longo período de incubação desta infecção, o perfil epidemiológico apresentado pelos casos, reflete um padrão de transmissão do vírus de 5 a 10 anos atrás ${ }^{54}$.

Mesmo entendendo que a notificação dos indivíduos HIV positivos não pode ser utilizada para cálculo de incidência/prevalência desta infecção na população, uma vez que se refere à demanda de serviços, o conhecimento da população de portadores assintomáticos do HIV permite avaliar algumas tendências da epidemia quanto às categorias de exposição, faixa etária, escolaridade, ocupação, residência, etc.

As informações do sistema de HIV positivos podem ser utilizadas na elaboração de estratégias mais eficazes de prevenção, além de permitir avaliações operacionais da demanda dos serviços e fornecer subsídios para uma melhor organização das atividades assistenciais e de planejamento.

Desde 1994 foi implantado no ESP o Sistema de Informação de Soropositivo Assintomático - SIHIV. E com a implantação do Sistema de Informação de Agravos de Notificação em sua versão para o Windows SINAN-W, em novembro de 2001, a Unidade de Epidemiologia da Coordenação Nacional de DST/aids e a Vigilância Epidemiológica do Programa Estadual de DST/aids do Estado de São Paulo recomendaram que todos os portadores assintomáticos do HIV passassem a ser notificados neste sistema, através da mesma ficha utilizada para a notificação dos casos de aids. A V.E. de DST/aids tem discutido com o Centro de Vigilância Epidemiológica "Alexandre Vranjac" e com outras instâncias da Secretaria de Estado da Saúde de São Paulo a possibilidade de se incluir a infecção pelo HIV no rol dos agravos de notificação compulsória neste Estado.

Ressalta-se que a notificação do HIV positivo não exclui a notificação compulsória do paciente, quando ele preenche os critérios de definição de caso de aids.

\section{Aprimoramento da investigação da categoria de exposição para os casos de HIV/aids.}

Diante de um diagnóstico de aids é difícil precisar o momento de ocorrência da infecção pelo HIV, dado o seu longo período de incubação. Assim, a Vigilância Epidemiológica de DST/aids do Programa Estadual SP, tem recomendado que se proceda à avaliação das possibilidades de risco para aquisição da infecção pelo HIV nos 10 últimos anos, exceção feita aos indivíduos que sabem precisar o período em que tiveram viragem sorológica, ou porque fazem o teste anti-HIV com uma certa periodicidade (como os doadores de sangue habituais) ou porque foram submetidos a um teste ELISA detuned, que permite avaliar se a infecção é recente (no último ano).

A avaliação de risco deve ser o mais isenta possível de valoração quanto aos aspectos morais e de comportamento. Por exemplo, uma mulher HIV positiva, que teve 2 parceiros nos últimos dez anos, sem outras exposições ao risco, e que não sabe com qual dos dois parceiros se infectou, vai ser classificada, para fins de categorização de risco, como heterossexual de múltiplos parceiros. Uma pessoa que tenha feito um uso eventual de drogas injetáveis 8 anos atrás, que não tem outra possibilidade conhecida de infecção pelo HIV e desenvolver um quadro de aids, vai ser classificada como usuária de drogas injetáveis. Ou seja, a categorização de risco para os casos de aids pode não refletir a orientação sexual ou o hábito de uso de drogas dos indivíduos pesquisados.

Com relação à transmissão por transfusão sangüínea, o que importa não é saber se o indivíduo recebeu transfusão ou não, mas se esta pode ser considerada o fator de risco para a aquisição do HIV. Portanto, toda vez que se detectar um possível fator de risco, 
deve ser considerado o nexo temporal entre a exposição de risco e a viragem sorológica e/ou a manifestação da aids. Este raciocínio se aplica a todas as categorias de exposição.

A investigação sobre a situação de risco dos casos de HIV/aids é muito importante e deve ser criteriosa, pois esta informação tem subsidiado a definição de grupos de risco acrescido e de maior vulnerabilidade, para os quais devem se voltar prioritariamente as atividades de prevenção e vigilância.

\section{O quesito raça/cor na investigação epidemiológica.}

O quesito cor é uma variável necessária, devendo ser adotado nos estudos e dados epidemiológicos sobre as DST/aids (e outras doenças) como um indicador da vulnerabilidade de diferentes grupos étnicos. Embora a medida, para alguns críticos, pareça 'racismo', ela possibilita a adoção de políticas públicas preventivas específicas e, portanto, mais eficazes.

Após muitos anos de reivindicação do movimento negro, o quesito cor/raça foi finalmente introduzido nos dados de identificação da ficha de notificação/investigação do SINAN-W, e o seu preenchimento deve ser feito preferencialmente pelo método de autoclassificação.

\section{A Vigilância de segunda geração do HIV/ aids.}

Com o intuito de sistematizar as discussões sobre as perspectivas da vigilância epidemiológica de HIV/aids, no ano 2000, a Organização Mundial de Saúde (OMS) e a UNAIDS elaboraram um documento denominado "Diretrizes para a Vigilância de Segunda Geração do HIV"55.

Os sistemas de vigilância de segunda geração têm por objetivo identificar, tanto as tendências do comportamento através de estudos transversais repetidos na população geral e em subgrupos da população que investiguem questões como freqüência e tipo de relações sexuais, uso de preservativos, idade na primeira relação sexual, uso de dro- gas injetáveis, uso compartilhado de seringas e outras, quanto as tendências da infecção do HIV através da vigilância biológica, tais como: vigilância sorológica sentinela em determinados subgrupos da população, detecção de rotina do HIV entre doadores de sangue, entre coortes de trabalhadores ou outros subgrupos da população, e em amostras biológicas obtidas por meio de pesquisas na população geral.

Os dados sobre comportamento ajudam a explicar as tendências da prevalência da infecção. Para se compreender totalmente a dinâmica da epidemia, é preciso saber quais são as pessoas que têm risco acrescido na região/município/estado/país e quais são os comportamentos que contribuem para esse risco, assim como identificar as populações mais vulneráveis à infecção pelo HIV, utilizando a informação gerada pela vigilância para melhorar o conhecimento da epidemia e elaborar planos de prevenção e assistência mais próximos da realidade.

Assim, as estratégias de prevenção e controle do HIV/aids devem ser voltadas prioritariamente para populações alvo, definidas a partir das populações de risco acrescido e de maior vulnerabilidade. Entende-se por populações de risco acrescido os grupos que por características ou comportamentos específicos têm maior probabilidade de se infectar pelo HIV, considerados segundo estes determinados aspectos, tendo redes de interação mais fechadas que formam grupos mais homogêneos, como por exemplo os usuários de drogas injetáveis e homens que fazem sexo com homens. As populações ou grupos que apresentam maior vulnerabilidade, são as que estabelecem redes sociais mais abertas e são mais heterogêneas em sua composição e que não necessariamente apresentam risco acrescido, mas que pela sua inserção na sociedade têm menor chance de proteção diante da epidemia de HIV/Aids, como por exemplo as mulheres e os índios.

Os princípios da vigilância de segunda geração consideram que os sistemas de vigilância devem ser os indicados para cada estágio da epidemia; ser dinâmicos o bastante 
para se adaptarem às mudanças da mesma; destinar os seus recursos de maneira a gerar a informação mais útil possível e comparar os dados biológicos com os dados comportamentais para obter o máximo potencial explicativo; e integrar rotineiramente a in- formação de outras fontes, como a vigilância dos casos de infecção por HIV, do sistema de mortalidade, da vigilância das DST, de medicamentos, da tuberculose e outros, e utilizar o produto dos dados para aumentar e aperfeiçoar a resposta à epidemia.

\section{Referências}

1. Centers for Disease Control and Prevention - CDC. Kaposis sarcoma and pneumocistis pneumonia among homossexual male residents of New York city and California. MMWR Morb Mortal Wkly Rep 1981; 30: 305-8.

2. Gottlieb MS, Schroff R, Schanker HM, et al. Pneumocystis Carinii pneumonia and mucosal cadidiasis in previously healthy homossexual men: evidence of a new acquired cellular immunodeficiency. N Engl J Med 1981; 305: 1425-31.

3. Centers for Disease Control and Prevention - CDC. First report of AIDS. MMWR Morb Mortal Wkly Rep 2001; 50: 429 .

4. Barré-Sinoussi F, Montagneir L, Chermann JC, Rey F, Nugyese MT. Isolation of T-lynphotropic retrovirus from a patient at risk for acquired immune deficiency syndrome (AIDS). Science 1983; 220: 868.

5. Gallo RC, Popovic M, et al. Frequent detection and isolation of cytopathic retrovirus (HTLV-III)from patientes with AIDS and a risk for AIDS. Science 1984; 224: 500-3.

6. Centers for Disease Control and Prevention - CDC. HIV and AIDS - United States, 1981-2000. MMWR Morb Mortal Wkly Rep 2001; 50(21): 430-3.

7. European Center for the Epidemiological Monitoring of AIDS. HIV/AIDS Surveillance in Europe: Report $n^{\circ}$ 61; 1999.

8. Brasil. Ministério da Saúde - Coordenação Nacional DST/Aids - Vigilância Epidemiológica. Boletim Epidemiológico de AIDS ano XIV nº 2 . Brasília; abril a junho de 2001.

9. Bastos FI, Barcellos C. Geografia social da AIDS no Brasil. Rev Saúde Pública 1995; 29(1): 52-62.

10. Centers for Disease Control and Prevention - CDC. The Global HIV and AIDS Epidemic,2001. MMWR Morb Mortal Wkly Rep 2001; 50: 434-9.

11. Mann J, Tarantola DJM, Netter TW. Como avaliar a vulnerabilidade à infecção pelo HIV e AIDS. In: Richard Parker, Jane Galvão, José Stalin Pedrosa (orgs da edição brasileira) - A AIDS no Mundo. Rio de Janeiro: Relumé Dumará: ABIA: IMS, UERJ; 1993. p. 275-300.
12. Piot P, Quinn TC, Taelman H, Minlangu KB et al. Acquired immunodeficiency syndrome in a heterossexual population in Zaire. Lancet 1984; 14: 659.

13. Secretaria de Estado da Saúde de SP - Programa Estadual DST/AIDS - Vigilância Epidemiológica Boletim Epidemiológico ano XIX - nº 1. São Paulo; abril, 2001.

14. Santos NJS. A Aids entre as mulheres no Estado de São Paulo. In: Parker R e Galvão J (orgs). Quebrando o silêncio: mulheres e Aids no Brasil. Rio de Janeiro: Relume-Dumará: ABIA:IMS/UERJ; 1996; parte 1, cap. 2: 33 .

15. Secretaria Municipal de Saúde de São Paulo Programa Municipal de DST/AIDS. Boletim Epidemiológico de AIDS ano I - nº1. São Paulo; 1997.

16. Centers for Disease Control and Prevention - CDC. A group of HIV positive women. MMWR Morb Mortal Wkly $\boldsymbol{R e p ~ 1 9 9 9 ; ~ 4 8 : ~ 4 1 3 - 6 . ~}$

17. Bastos, FI. Ruína e construção: AIDS e drogas injetáveis na cena contemporânea. Rio de Janeiro: RelumeDumará/ABIA/IMS-UERJ; 1996.(História Social da AIDS, 6).

18. Kalichman AO. Pauperização e banalização de uma epidemia. In: Seminário Epidemiologia Social da AIDS, Anais. Rio de Janeiro; 1994. p. 20-6.

19. Parker R, Camargo Jr KR. Pobreza e HIV/AIDS: aspectos antropológicos e sociológicos. Cad Saúde Pública 2000; 16(Supll 1): 89-102.

20. Szwarcwald CL, Bastos FI. AIDS e Pauperização: Principais conceitos e evidências empíricas. In: Sobre a epidemia da aids no Brasil: distintas abordagens. Brasília: Ministério da Saúde, Coordenação Nacional de DST e AIDS; 1999. p. 7-19.

21. Bastos, FI. A feminização da epidemia de Aids no Brasil: Determinantes Estruturais e Alternativas de enfrentamento. In: Saúde Sexual e Reprodutiva, no 3 . Rio de Janeiro: ABIA/IMS-UERJ; 2000. (Coleção ABIA)

22. Moore JS, Harrison JS, Doll LS. Interventions for Sexually Active, Heterosexual Women in the United States. In: Di Clemente RJ, Peterson JL (edit). Preventing AIDS: Theories and Methods of Behavioral Interventions. New York: Plenum Press, 1994; p. 243-62. 
23. Quinn SC. AIDS and the African American Woman: The triple burden of race, class and gender. Health

Education Quarterly 1993. Vol. 20(3): 305-20.

24. Lira MMTA. Mortalidade Prematura no Município de São Paulo. Anos Potenciais de Vida Perdidos: 1980, 1985, 1990 e 1995. São Paulo; 1998.[Dissertação de Mestrado - Faculdade de Saúde Pública da USP].

25. Joint United Nations Programme on HIV/AIDS (UNAIDS). El primer análises de la epidemia mundial, país por país, revela inquietantes diferencias com respecto al SIDA. Geneva: UNAIDS; 1998.

26. Lei N. ${ }^{\circ} 7.649$ de 25 de janeiro de 1988. Estabelece a obrigatoriedade de cadastramento dos doadores de sangue, bem como a realização de exames laboratoriais no sangue coletado, visando a prevenir a propagação de doenças, e dá outras providências. Pub. D.O.U. de $27 / 01 / 88$

27. Brasil. Ministério da Saúde - Coordenação Nacional DST/Aids - Vigilância Epidemiológica. Boletim Epidemiológico de AIDS ano XIV - n 2. Brasília; abril a junho de 2001.

28. Szwarcwald CL, Carvalho MF. Estimativa do número de indivíduos de 15 a 49 anos infectados pelo HIV, Brasil, 2000. Boletim Epidemiológico de Aids, ano XIV, no 1 , janeiro a março de 2001.

29. Brasil, Ministério da Saúde, Coordenação Nacional DST/AIDS. Revisão da definição nacional de casos de aids em indivíduos com 13 anos ou mais, para fins de vigilância epidemiológica. MS. Secretaria de politicas de saude, coordenação nacional de DST e aids, Brasilia, 1998.

30. Brasil, Ministério da Saúde, Coordenação Nacional DST/AIDS. Revisão da definição nacional de casos de aids em indivíduos menores de 13 anos, para fins de vigilancia epidemiologica. MS Secretaria de politicas de saude, coordenação nacional de DST e aids, Brasilia, 2000 .

31. Chaisson RE. Impact of opportunistic disease on survival in patients with HIV infection. AIDS. Aids 2001. Vol. 12(1): 29-33.

32. Rao VK. The impact of comorbidity on mortalidade following in-hospital diagnosis of tuberculosis. Chest 1998; 114(5): 1244-52.

33. Bower JE; Kemeny ME; Taylor SE; Fahey JL. Cognitive processing, discovery of meaning, CD4 decline, and Aids related mortality among bereaved HIVseropositive men. J Consult Clin Psychol 1998; 66(6): 979-86.

34. Moatti JP, Souteyrand Y. HIV/AIDS social and behavioural research: past advances and thoughts about the future. Social Sci Med 2000; 50: 1519-32.

35. Greco DB. Ética, Saúde e Pobreza - As doenças emergentes no século XXI. Bioética 1999; 7: 189-98.
36. Lei N. ${ }^{\circ} 5.190$ de 20 de junho de 1986. Dispõe sobre a realização de testes para detecção de anticorpos do vírus da Síndrome da Deficiência Imunológica Adquirida (AIDS). Pub. D.O.E. de 21/06/86.

37. Parker R. A construção da solidariedade: AIDS, sexualidade e política no Brasil. Rio de Janeiro, Relume-Dumará/ABIA/IMS-UERJ; 1994. Cap. 3. Abaixo do Equador: bissexualidade e AIDS no Brasil. p. 55-66. (História Social da AIDS, 3).

38. Parker R. Sexo entre homens: Consciência da AIDS e comportamento Sexual entre os homens homossexuais e bissexuais no Brasil. In: Parker R. et al [Orgs]. A Aids no Brasil. Rio de Janeiro: Relume - Dumará; 1994. p. 129-49.

39. Buchalla CM. A Síndrome da Imunodeficiência Adquirida e a mortalidade masculina, de 20 a 49 anos, no Município de São Paulo:1983 a 1986. [tese de doutorado]. São Paulo; Faculdade de Saúde Pública da USP; 1991.

40. Waldvogel B, Morais LCC. Mortalidade por AIDS em São Paulo: dezoito anos de história. In: Secretaria de Estado da Saúde de SP - Programa Estadual DST/Aids - Vigilância Epidemiológica - Boletim Epidemiológico ano XVI - no 2. São Paulo; 1998.

41. Waldvogel B, Morais LCC. O cenário promissor da evolução da mortalidade por AIDS no Estado de São Paulo no final do século XX. In: Secretaria de Estado da Saúde de SP - Programa Estadual DST/Aids - Vigilância Epidemiológica -. Boletim Epidemiológico ano XX - nº 1 . São Paulo; 2002.

42. Grangeiro A. O perfil sócio econômico dos casos de aids da cidade de São Paulo. In: Parker R et al [Orgs]. A Aids no Brasil. Rio de Janeiro: Relume - Dumará, 1994. p. 91-125.

43. Fonseca MG, Bastos FI, Derrico M, Andrade CLT et al. AIDS e grau de escolaridade no Brasil: evolução temporal de 1986 a 1996. Cad Saúde Pública 2000; 16(Supl 1): 77-87.

44. Badiani R, Quental I, Santos EM. DST/AIDS e a Pesquisa Nacional sobre Demografia e Saúde: Uma análise do nível de conhecimento e comportamentos de vulnerabilização. Relatório de pesquisa - BENFAM e DHS. Rio de Janeiro; 1997.

45. Plummer FA, Moses S, Ndinya-Achola JO. Factors affecting female-to-male transmission of HIV: Implications of transmission dynamics for prevention. In: Chen L.C. et al (Ed.). AIDS and Women's Reproductive Health. Plenum Press, New York, 1991.

46. Downs AM, Vicenzi I . Probability of Heterosexual Transmission of HIV: Relationship to the number of unprotected sexual contacts. Journal of AIDS and Human Retrovirology 1996; 11: 388-95.

47. Ueli Zelhveger J, Wang M R, Schrid B. The male IDU epidemic is closely linked to its secondary epidemic among heterossexual women in Europe. 12th World AIDS Conference. Geneva; 1998. (Anais). 
48. Greco DU. Epidemiological profile of women over age 15 at risk or infected with HIV in Belo Horizonte, Brazil - Jan.-Dec. 1996. 12th World AIDS Conference. Geneva; 1998. (Anais).

49. Martins TA, Queiroz TRB, Broutet N, Evangelista CN, Feitosa IS. Profile of the women with AIDS and the women consulted at STD clinics in Fortaleza, Ceara, Northeast Brazil. 12th World AIDS Conference. Geneva; 1998. (Anais).

50. Vermelho L. Women with AIDS: transmission and prevention strategies. 12th World AIDS Conference. Geneva; 1998 (Anais).

51. Ministério da Saúde - Coordenação Nacional DST/Aids e CEBRAP - Centro Brasileiro de Análise e Planejamento. Comportamento sexual da população brasileira e percepções do HIV/AIDS. Brasília; 2000.
52. Giffin K. Beyond empowerment: heterosexualities and the prevention of AIDS. Soc Sci Med 1998; 46(2): 1516.

53. Szwarcwald CL, Andrade CLT, Castilho EA. Estimativa do número de órfãos decorrentes da AIDS materna, Brasil, 1987-1999. Cad Saúde Pública 2000; 16(Supl 1): 129-34.

54. Secretaria de Estado da Saúde de São Paulo - Manual de Vigilância Epidemiológica da AIDS - Vigilância Epidemiológica - Programa Estadual DST/AIDS - 1995.

55. ONUSIDA/OMS .Guias sobre la vigilância del VIH de segunda generacíon - Grupo de trabajo de ONUSIDA/ OMS para la Vigilância Mundial Del VIH/SIDA e ITS; 2000 .

Recebido em 06/09/02; aprovado em 18/11/02 